\title{
Modelling One-Pot Method for Synthesis of 2,3-Dihydro-1H-pyrrolo[2,1-c][1,4]benzothiazine 5,5-Dioxides and Their Homologues
}

\author{
Oleksandr Yu Grevtsov, Oleg V. Zaremba, Anna B. Bondarenko, Oleksandr G. Drushlyak*, \\ Sergiy M. Kovalenko, Valentyn P. Chernykh \\ National University of Pharmacy, Kharkiv, Ukraine \\ Email: *aldry18@hotmail.com
}

Received March 25, 2013; revised May 8, 2013; accepted May 22, 2013

Copyright (C) 2013 Oleksandr Yu Grevtsov et al. This is an open access article distributed under the Creative Commons Attribution License, which permits unrestricted use, distribution, and reproduction in any medium, provided the original work is properly cited.

\begin{abstract}
A facile method for synthesis of 2,3-dihydro- $1 H$-pyrrolo[2,1-c][1,4]benzothiazines by interaction of methylenactive (2-fluorophenyl)sulfones with homologues of either 5-methoxy-3,4-dihydro-2H-pyrrole or 5-(methylthio)-3,4-dihydro$2 \mathrm{H}$-pyrrole has been developed.
\end{abstract}

Keywords: Sulfones; Lactims; Cyclization; Heterocycles; Nucleophilic Aromatic Substitution

\section{Introduction}

In recent years a substantial number of 1,1-dioxo- $4 H$ 1,4-benzothiazines have been reported to possess pharmacological activity. They were mentioned as glycineNMDA receptor antagonists [1] and protein kinase inhibitors, which can be used to treat cancer and hyperproliferative disorders [2]. Similar compounds were patented as potent antivirals [3], potassium channel openers [4], antiischemics to cure heart diseases [5], 3-hydroxy3-methylglutaryl-CoA reductase inhibitors [6], some of them were reported to be diuretics [7]. Also they were known as highly effective antimicrobial agents against Streptococcus and Klebsiella [8].

1,1-Dioxo-4H-1,4-benzothiazines can be readily synthesized by the oxidation of 4H-1,4-benzothiazines [913], intramolecular cyclization of $N$-[(2-alkylsulfonyl)phenyl]amides of carboxylic acids [14-17], spontaneous cyclization of 1-[(2-nitrophenyl)sulfonyl]ketones during reduction $[18,19]$.

The promising approach is also the cyclization of 2-[(2-halogenophenyl)sulfonyl] ethylenamines containing electronwithdrawing group $\mathrm{A}$ in the presence of bases [20-26] (Figure 1).

The last synthetic approach for 1,1-dioxo-4H-1,4benzothiadiazines is the most convenient for the achievement of high range molecular diversity due to the

"Corresponding author. variability of radicals in the aromatic ring $\left(\mathrm{R}^{1}\right)$ and the positions 2, 3, 4 of 1,4-benzothiadiazine moiety $\left(A, R^{3}\right.$, $R^{2}$ ). The most significant limitation of this method is mainly concerned with the leaving halogen (X) activity. For instance, the cyclization of orto-chloroderivatives (X $=\mathrm{Cl}$ ) could be successfully carried out only in the presence of strong bases [20], silver nitrate [22,23], potassium carbonate-crown-ether [21] or requires the application of microwave technology $[24,25]$. In the case of fluoroderivatives $(\mathrm{X}=\mathrm{F})$, the cyclization proceeds readily [26].

Though the cyclization of 2-[(2-halogenophenyl)sulfonyl] ethylenamines is a versatile methodology for 1,1dioxo-4H-1,4-benzothiadiazines obtaining, the synthesis of the heterocyclic systems where $\mathrm{R}^{2}$ and $\mathrm{R}^{3}$ are the parts of the same cycle was not reported yet.

The purpose of this paper is to develop the convenient synthetic way for 2,3-dihydro- $1 H$-pyrrolo[2,1-c][1,4]benzothiazine 5,5-dioxides and their homologues, which could be interesting objects for further pharmacological

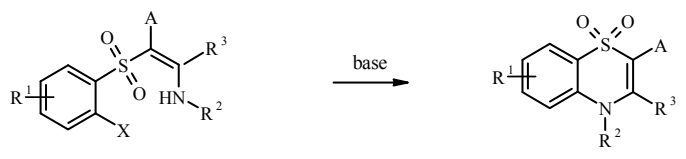

$\mathrm{R}^{1}=\mathrm{H}, \mathrm{Hal} ; \mathrm{R}^{2}=$ Alkyl, Aryl; $\mathrm{R}^{3}=\mathrm{H}$, Alkyl, SAlkyl, $\mathrm{NAlkyl}_{2} \mathrm{~A}=\mathrm{CN}$, COOAlkyl, Acyl; X = F, Cl

Figure 1. Cyclization of 2-[(2-halogenophenyl)sulfonyl]ethylenamines. 
screening.

It was reported that the interaction of asymmetrical methylene active compounds with either 5-methoxy3,4-dihydro-2H-pyrrole or 5-(methylthio)-3,4-dihydro$2 \mathrm{H}$-pyrrole and their homologues led to formation of mixture E,Z-isomers of enamine-type products [27-29]. With regard to this fact, the interaction between (2fluorophenyl)sulfones 1a-i and lactims 2a-f (Figure 2) should produce $2-\{[(2-f l u o r o p h e n y l)$ sulfonyl $]$ methylene $\}$ pyrrolidines and their homologues $3 \mathrm{a}-\mathrm{z}$ (Figure 1).

The heating of sulfones 1 with excess of lactims 2 (30\% for sulfonylacetonitriles $1 \mathrm{a}, \mathrm{d}-\mathrm{i}$ and $50 \%$ for other compounds 1) at $90^{\circ} \mathrm{C}$ has been chosen as the standard reaction conditions. The reaction for acetonitriles $1 \mathrm{a}, \mathrm{d}-\mathrm{i}$ was held in DMF media, for other compounds 1 was carried out solvent-free. The process was monitored by TLC and disappearance of starting sulfone 1 spot has been controlled. The results of experiment demonstrated that the interaction of sulfones 1 with 5-methoxy-3,4-dihydro- $2 H$-pyrrole and its homologues $2 \mathrm{a}-\mathrm{c}$ in chosen conditions did not produces solely products 3 . In some cases the products of further cyclization 4 were also present in the reaction mixture. The lactims 2 having larger cycle required more reaction time. Experimental data (Table 1)

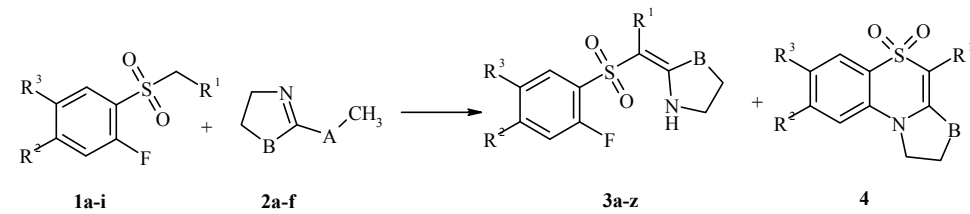

1a: $\mathrm{R}^{1}=\mathrm{CN}, \mathrm{R}^{2}=\mathrm{R}^{3}=\mathrm{H} ; \mathbf{1 b}: \mathrm{R}^{1}=\mathrm{Ac}, \mathrm{R}^{2}=\mathrm{R}^{3}=\mathrm{H} ; \mathbf{1 c}: \mathrm{R}^{1}=\mathrm{COOMe}, \mathrm{R}^{2}=\mathrm{R}^{3}=\mathrm{H} ; \mathbf{1 d}: \mathrm{R}^{1}=\mathrm{CN}, \mathrm{R}^{2}=\mathrm{H}, \mathrm{R}^{3}=\mathrm{Me}$; 1e: $R^{1}=C N, R^{2}=H, R^{3}=F ; 1$ f: $R^{1}=C N, R^{2}=H, R^{3}=C l ; 1$ g: $R^{1}=C N, R^{2}=M e, R^{3}=H ; 1$ h: $R^{1}=C^{2}, R^{2}=F, R^{3}=H$; 1i: $\mathrm{R}^{1}=\mathrm{CN}, \mathrm{R}^{2}=\mathrm{Cl}, \mathrm{R}^{3}=\mathrm{H} ; \mathbf{2} \mathbf{a}: \mathrm{A}=\mathrm{O}, \mathrm{B}=\mathrm{CH}_{2} ; \mathbf{2 b}: \mathrm{A}=\mathrm{O}, \mathrm{B}=\left(\mathrm{CH}_{2}\right)_{2} ; \mathbf{2}: \mathrm{A}=\mathrm{O}, \mathrm{B}=\left(\mathrm{CH}_{2}\right)_{3}$;

2d: $\mathrm{A}=\mathrm{S}, \mathrm{B}=\mathrm{CH}_{2} ; \mathbf{2 e}: \mathrm{A}=\mathrm{S}, \mathrm{B}=\left(\mathrm{CH}_{2}\right)_{2} ; \mathbf{2 f}: \mathrm{A}=\mathrm{S}, \mathrm{B}=\left(\mathrm{CH}_{2}\right)_{3}$.

Figure 2. Interaction of sulfones 1 with lactims 2.

Table 1. 2-\{[(2-Fluorophenyl)sulfonyl]methylene\}pyrrolidines and their homologues 3a-z.

\begin{tabular}{|c|c|c|c|c|c|c|c|c|c|}
\hline Cmpd. & Formula & M.W. & $\mathrm{A}$ & $\mathrm{B}$ & $\mathrm{R}^{1}$ & $\mathrm{R}^{2}$ & $\mathrm{R}^{3}$ & Time, $\mathrm{h}$ & Yield $3(4), \%$ \\
\hline $3 \mathbf{a}$ & $\mathrm{C}_{12} \mathrm{H}_{11} \mathrm{FN}_{2} \mathrm{O}_{2} \mathrm{~S}$ & 266.29 & $\mathrm{O}$ & $\mathrm{CH}_{2}$ & $\mathrm{CN}$ & $\mathrm{H}$ & $\mathrm{H}$ & 4 & 82 \\
\hline $\mathbf{3 b}$ & $\mathrm{C}_{13} \mathrm{H}_{13} \mathrm{FN}_{2} \mathrm{O}_{2} \mathrm{~S}$ & 280.32 & $\mathrm{O}$ & $\left(\mathrm{CH}_{2}\right)_{2}$ & $\mathrm{CN}$ & $\mathrm{H}$ & $\mathrm{H}$ & 4 & $72(14)$ \\
\hline $3 c$ & $\mathrm{C}_{14} \mathrm{H}_{15} \mathrm{FN}_{2} \mathrm{O}_{2} \mathrm{~S}$ & 294.34 & $\mathrm{O}$ & $\left(\mathrm{CH}_{2}\right)_{3}$ & $\mathrm{CN}$ & $\mathrm{H}$ & $\mathrm{H}$ & 8 & $54(18)$ \\
\hline 3d & $\mathrm{C}_{13} \mathrm{H}_{14} \mathrm{FNO}_{3} \mathrm{~S}$ & 283.32 & $\mathrm{O}$ & $\mathrm{CH}_{2}$ & Ac & $\mathrm{H}$ & $\mathrm{H}$ & 10 & 73 \\
\hline 3d & $\mathrm{C}_{13} \mathrm{H}_{14} \mathrm{FNO}_{3} \mathrm{~S}$ & 283.32 & $\mathrm{~S}$ & $\mathrm{CH}_{2}$ & Ac & $\mathrm{H}$ & $\mathrm{H}$ & 4 & 80 \\
\hline $3 e$ & $\mathrm{C}_{14} \mathrm{H}_{16} \mathrm{FNO}_{3} \mathrm{~S}$ & 297.35 & $\mathrm{~S}$ & $\left(\mathrm{CH}_{2}\right)_{2}$ & Ac & $\mathrm{H}$ & $\mathrm{H}$ & 6 & 31 \\
\hline $3 f$ & $\mathrm{C}_{13} \mathrm{H}_{14} \mathrm{FNO}_{4} \mathrm{~S}$ & 299.32 & $\mathrm{O}$ & $\mathrm{CH}_{2}$ & $\mathrm{CO}_{2} \mathrm{Me}$ & $\mathrm{H}$ & $\mathrm{H}$ & 10 & 57 \\
\hline $3 f$ & $\mathrm{C}_{13} \mathrm{H}_{14} \mathrm{FNO}_{4} \mathrm{~S}$ & 299.32 & $\mathrm{~S}$ & $\mathrm{CH}_{2}$ & $\mathrm{CO}_{2} \mathrm{Me}$ & $\mathrm{H}$ & $\mathrm{H}$ & 10 & 85 \\
\hline $3 g$ & $\mathrm{C}_{14} \mathrm{H}_{16} \mathrm{FNO}_{4} \mathrm{~S}$ & 313.36 & $\mathrm{~S}$ & $\left(\mathrm{CH}_{2}\right)_{2}$ & $\mathrm{CO}_{2} \mathrm{Me}$ & $\mathrm{H}$ & $\mathrm{H}$ & 20 & 42 \\
\hline $3 \mathbf{h}$ & $\mathrm{C}_{15} \mathrm{H}_{18} \mathrm{FNO}_{4} \mathrm{~S}$ & 327.37 & $\mathrm{~S}$ & $\left(\mathrm{CH}_{2}\right)_{3}$ & $\mathrm{CO}_{2} \mathrm{Me}$ & $\mathrm{H}$ & $\mathrm{H}$ & 20 & 48 \\
\hline $3 \mathbf{i}$ & $\mathrm{C}_{13} \mathrm{H}_{13} \mathrm{FN}_{2} \mathrm{O}_{2} \mathrm{~S}$ & 280.32 & $\mathrm{O}$ & $\mathrm{CH}_{2}$ & $\mathrm{CN}$ & $\mathrm{H}$ & $\mathrm{Me}$ & 4 & 92 \\
\hline $3 \mathbf{j}$ & $\mathrm{C}_{12} \mathrm{H}_{10} \mathrm{~F}_{2} \mathrm{~N}_{2} \mathrm{O}_{2} \mathrm{~S}$ & 284.28 & $\mathrm{O}$ & $\mathrm{CH}_{2}$ & $\mathrm{CN}$ & $\mathrm{H}$ & $\mathrm{F}$ & 4 & $84(7)$ \\
\hline 3k & $\mathrm{C}_{12} \mathrm{H}_{10} \mathrm{ClFN}_{2} \mathrm{O}_{2} \mathrm{~S}$ & 300.74 & $\mathrm{O}$ & $\mathrm{CH}_{2}$ & $\mathrm{CN}$ & $\mathrm{H}$ & $\mathrm{Cl}$ & 4 & $76(13)$ \\
\hline 31 & $\mathrm{C}_{13} \mathrm{H}_{13} \mathrm{FN}_{2} \mathrm{O}_{2} \mathrm{~S}$ & 280.32 & $\mathrm{O}$ & $\mathrm{CH}_{2}$ & $\mathrm{CN}$ & $\mathrm{Me}$ & $\mathrm{H}$ & 4 & 96 \\
\hline $3 m$ & $\mathrm{C}_{12} \mathrm{H}_{10} \mathrm{~F}_{2} \mathrm{~N}_{2} \mathrm{O}_{2} \mathrm{~S}$ & 284.28 & $\mathrm{O}$ & $\mathrm{CH}_{2}$ & $\mathrm{CN}$ & $\mathrm{F}$ & $\mathrm{H}$ & 4 & $32(62)$ \\
\hline $3 n$ & $\mathrm{C}_{12} \mathrm{H}_{10} \mathrm{ClFN}_{2} \mathrm{O}_{2} \mathrm{~S}$ & 300.74 & $\mathrm{O}$ & $\mathrm{CH}_{2}$ & $\mathrm{CN}$ & $\mathrm{Cl}$ & $\mathrm{H}$ & 4 & $81(9)$ \\
\hline 30 & $\mathrm{C}_{14} \mathrm{H}_{15} \mathrm{FN}_{2} \mathrm{O}_{2} \mathrm{~S}$ & 294.34 & $\mathrm{O}$ & $\left(\mathrm{CH}_{2}\right)_{2}$ & $\mathrm{CN}$ & $\mathrm{H}$ & $\mathrm{Me}$ & 4 & 89 \\
\hline $3 \mathbf{p}$ & $\mathrm{C}_{13} \mathrm{H}_{12} \mathrm{~F}_{2} \mathrm{~N}_{2} \mathrm{O}_{2} \mathrm{~S}$ & 298.31 & $\mathrm{O}$ & $\left(\mathrm{CH}_{2}\right)_{2}$ & $\mathrm{CN}$ & $\mathrm{H}$ & $\mathrm{F}$ & 4 & $60(16)$ \\
\hline $3 q$ & $\mathrm{C}_{13} \mathrm{H}_{12} \mathrm{ClFN}_{2} \mathrm{O}_{2} \mathrm{~S}$ & 314.76 & $\mathrm{O}$ & $\left(\mathrm{CH}_{2}\right)_{2}$ & $\mathrm{CN}$ & $\mathrm{H}$ & $\mathrm{Cl}$ & 4 & $45(45)$ \\
\hline $3 \mathbf{r}$ & $\mathrm{C}_{14} \mathrm{H}_{15} \mathrm{FN}_{2} \mathrm{O}_{2} \mathrm{~S}$ & 294.34 & $\mathrm{O}$ & $\left(\mathrm{CH}_{2}\right)_{2}$ & $\mathrm{CN}$ & $\mathrm{Me}$ & $\mathrm{H}$ & 4 & 94 \\
\hline $3 s$ & $\mathrm{C}_{13} \mathrm{H}_{12} \mathrm{~F}_{2} \mathrm{~N}_{2} \mathrm{O}_{2} \mathrm{~S}$ & 298.31 & $\mathrm{O}$ & $\left(\mathrm{CH}_{2}\right)_{2}$ & $\mathrm{CN}$ & $\mathrm{F}$ & $\mathrm{H}$ & 4 & $18(74)$ \\
\hline $3 t$ & $\mathrm{C}_{13} \mathrm{H}_{12} \mathrm{ClFN}_{2} \mathrm{O}_{2} \mathrm{~S}$ & 314.76 & $\mathrm{O}$ & $\left(\mathrm{CH}_{2}\right)_{2}$ & $\mathrm{CN}$ & $\mathrm{Cl}$ & $\mathrm{H}$ & 4 & $50(42)$ \\
\hline $3 \mathbf{u}$ & $\mathrm{C}_{15} \mathrm{H}_{17} \mathrm{FN}_{2} \mathrm{O}_{2} \mathrm{~S}$ & 308.37 & $\mathrm{O}$ & $\left(\mathrm{CH}_{2}\right)_{3}$ & $\mathrm{CN}$ & $\mathrm{H}$ & $\mathrm{Me}$ & 8 & 76 \\
\hline $3 \mathbf{v}$ & $\mathrm{C}_{14} \mathrm{H}_{14} \mathrm{~F}_{2} \mathrm{~N}_{2} \mathrm{O}_{2} \mathrm{~S}$ & 312.34 & $\mathrm{O}$ & $\left(\mathrm{CH}_{2}\right)_{3}$ & $\mathrm{CN}$ & $\mathrm{H}$ & $\mathrm{F}$ & 8 & $48(26)$ \\
\hline $3 \mathbf{w}$ & $\mathrm{C}_{14} \mathrm{H}_{14} \mathrm{ClFN}_{2} \mathrm{O}_{2} \mathrm{~S}$ & 328.79 & $\mathrm{O}$ & $\left(\mathrm{CH}_{2}\right)_{3}$ & $\mathrm{CN}$ & $\mathrm{H}$ & $\mathrm{Cl}$ & 8 & $49(9)$ \\
\hline $3 x$ & $\mathrm{C}_{15} \mathrm{H}_{17} \mathrm{FN}_{2} \mathrm{O}_{2} \mathrm{~S}$ & 308.37 & $\mathrm{O}$ & $\left(\mathrm{CH}_{2}\right)_{3}$ & $\mathrm{CN}$ & $\mathrm{Me}$ & $\mathrm{H}$ & 8 & $64(16)$ \\
\hline $3 y$ & $\mathrm{C}_{14} \mathrm{H}_{14} \mathrm{~F}_{2} \mathrm{~N}_{2} \mathrm{O}_{2} \mathrm{~S}$ & 312.34 & $\mathrm{O}$ & $\left(\mathrm{CH}_{2}\right)_{3}$ & $\mathrm{CN}$ & $\mathrm{F}$ & $\mathrm{H}$ & 8 & $0(73)$ \\
\hline $3 z$ & $\mathrm{C}_{14} \mathrm{H}_{14} \mathrm{ClFN}_{2} \mathrm{O}_{2} \mathrm{~S}$ & 328.79 & $\mathrm{O}$ & $\left(\mathrm{CH}_{2}\right)_{3}$ & $\mathrm{CN}$ & $\mathrm{Cl}$ & $\mathrm{H}$ & 8 & $0(53)$ \\
\hline
\end{tabular}


show that the compounds 3 with $\mathrm{R}^{1}=\mathrm{CN}$ are the most susceptible for cyclization. Thus, they were chosen as the model objects to study the influence of the substituent in benzene ring on the cyclization of compounds 3. It was established that electrondonating groups interdict formation of 1,4-benzothiadiazine ring, at the same time electronwithdrawing groups (halogens) promote cyclization of enamines 3. For example, the combination of O-methyllactim $2 \mathrm{c}$ with sulfonylacetonitriles $1 \mathrm{~h}$, $\mathrm{i}$, where $\mathrm{R}^{2}=$ $\mathrm{Cl}$ and $\mathrm{F}$, only gave the product of cyclization 8,9, 10,11-tetrahydro-7H-azepino[2,1-c][1,4]benzothiazine-6carbonitrile 5,5-dioxides $4 y$, z. The reaction of sulfonylacetone $1 \mathrm{~b}\left(\mathrm{R}^{1}=\mathrm{Ac}\right)$ with O-methyllactim 2 a resulted in selectively (1E)-1-[(2-fluorophenyl)sulfonyl]-1-(pyrrolidin-2-ylidene)acetone $3 \mathrm{~d}$, and its interaction with lactims $2 \mathrm{~b}$ and $2 \mathrm{c}$ allowed us to isolate only 2-methyl1,4-benzoxathiine 4,4-dioxide 5 (Figure 3). Probably in the case of sterically hindered lactims $2 b$, c, the competing reaction of intramolecular cyclization to 2-methyl1,4-benzoxathiine-4,4-dioxide 5 became the preferable process.

To prove the formation of compound 5 in this reaction, we performed its alternative synthesis by heating of sulfonylacetone $1 \mathrm{~b}$ in 1,4-dioxane at $90^{\circ} \mathrm{C}$ in the presence of equimolar amount DBU for 4 hours.

The reaction of sulfonylacetone $1 \mathrm{~b}\left(\mathrm{R}^{1}=\mathrm{Ac}\right)$ with S-methyllactims $2 \mathrm{~d}$ and $2 \mathrm{e}$ lead to formations of enamine type products $3 \mathrm{~d}$ and $3 \mathrm{e}$, and S-methyllactim $2 \mathrm{f}(2 \mathrm{Z})-2$ $\{[(2$-fluorophenyl)sulfonyl]methylidene $\}$ azepane 6 has been isolated with the yield $25 \%$ as mixture of $E$ - and $Z$ isomers.

The interaction of methyl [(2-fluorophenyl)sulfonyl]acetate $1 \mathrm{c}$ with $\mathrm{O}$-methyllactim $2 \mathrm{a}$ requires more time than interaction with sulfones $1 \mathrm{a}$ and $1 \mathrm{~b}$ and results in the mixture of $E$ - and $Z$ - isomers of enamine 3 f. The reaction of sulfone $1 \mathrm{c}$ with larger O-methyllactims $2 \mathrm{~b}, \mathrm{c}$ is failed.

The only reaction of methyl [(2-fluorophenyl)sulfonyl]acetate $1 \mathrm{c}$ with S-methyllactims $2 \mathrm{~d}-\mathrm{f}$ allowed us to obtain methyl (2E,Z)-[(2-fluorophenyl)sulfonyl](pyrrolidin-2ylidene)acetate $3 \mathrm{f}$ and its homologues $3 \mathrm{~g}$ and $3 \mathrm{~h}$ with

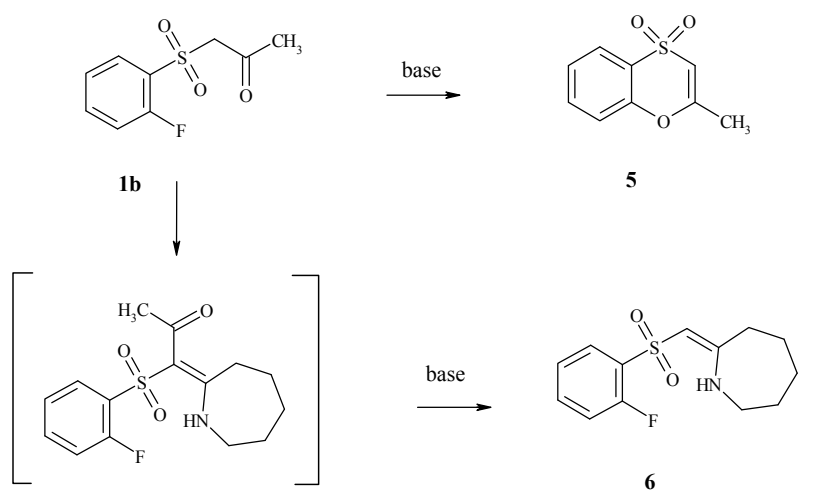

Figure 3. Transformations of sulfonylacetone $\mathbf{1 b}$. moderate yields.

The cyclization of enamines 3 to 2,3-dihydro- $1 H$ pyrrolo[2,1-c][1,4]benzothiazine 5,5-dioxides and their homologues 4 has been performed by heating in 1,4ioxane with equimolar amount of DBU at $50^{\circ} \mathrm{C}-60^{\circ} \mathrm{C}$ according to Figure 4.

Since 2-fluorophenylsulfones 1 interaction with O- or S-methyllactims 2 was a satisfactory approach for enamines 3 , and further cyclization of the condensation products 3 to the 2,3-dihydro- $1 H$-pyrrolo[2,1-c][1,4]benothiazine 5,5-dioxides and their homologues 4 occurred in numerous cases, it was interesting to develop "one-pot" method for synthesis of the products 4. For this purpose O-methyllactims 2a-c were chosen as the starting material for interaction with [(2-fluorophenyl)sulfonyl]acetonitriles 1a, d-i, S-methyllactims $2 \mathrm{~d}-\mathrm{f}$ were used for condensation with 1-[(2-fluorophenyl)sulfonyl] acetone $1 \mathrm{~b}$ and methyl [(2-fluorophenyl)sulfonyl] acetate $1 \mathrm{c}$.

According to the proposed "one-pot", procedure enamines 3 were not isolate. When the reaction of sulfones 1 with lactims 2 was complete, to the cool reaction mixture $\left(20^{\circ} \mathrm{C}\right)$ 1,4-dioxane and equimolar amount of DBU were added and the reaction mixture was heated at $60^{\circ} \mathrm{C}$ for $2-4$ hours. After dilution of reaction mixture with 2-propanol, the precipitate formed was filtered and crystallized from DMF-2-propanol mixture. The yields and some properties of obtained compounds are given in Table 2.

\section{Experimental Section}

The melting points $\left({ }^{\circ} \mathrm{C}\right)$ were measured with a Buchi B-520 melting point apparatus and were not corrected. IR spectra were recorded on FT-IR Bruker Tensor-27 spectrometer in $\mathrm{KBr}$. Thin-layer chromatography (TLC) was performed on aluminum sheets precoated with silica gel (Merck, Kieselgel 60 F-254). LC/MS spectra were recorded with PE SCIEX API 150EX liquid chromatograph equipped with a UV detector ( $\lambda_{\max } 215$ and $254 \mathrm{~nm}$ ) and using a $\mathrm{C}_{18}$ column $(100 \times 4 \mathrm{~mm})$. Elution started with water and ended with acetonitrile/water $(95: 5, \mathrm{v} / \mathrm{v})$ and used a linear gradient at a flow rate of $0.15 \mathrm{~mL} / \mathrm{min}$ and an analysis cycle time of $25 \mathrm{~min} .{ }^{1} \mathrm{H}$ NMR-spectra were

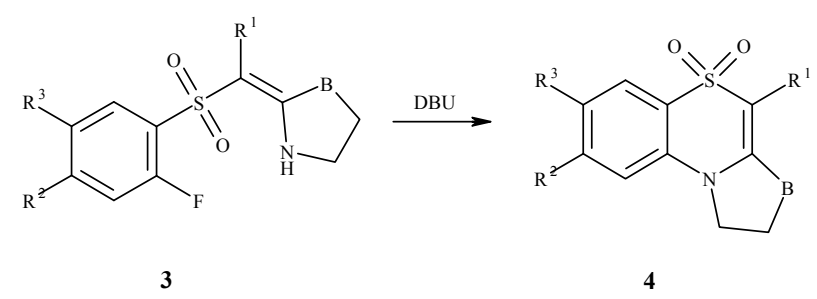

Figure 4. Cyclization of enamines 3 to 2,3-dihydro-1Hpyrrolo[2,1-c][1,4]benzothiazine 5,5-dioxides and their homologues 4 . 
Table 2. 2,3-Dihydro-1H-pyrrolo[2,1-c][1,4]benzothiazine 5,5-dioxides and their homologues 4 .

\begin{tabular}{|c|c|c|c|c|c|c|c|c|c|c|}
\hline Cmpd. & Formula & M.w. & $\mathrm{A}$ & B & $\mathrm{R}^{1}$ & $\mathrm{R}^{2}$ & $\mathrm{R}^{3}$ & Yield (two-steps), \% & Yield (one-pot), \% & m.p., ${ }^{\circ} \mathrm{C}$ \\
\hline $4 a$ & $\mathrm{C}_{12} \mathrm{H}_{10} \mathrm{~N}_{2} \mathrm{O}_{2} \mathrm{~S}$ & 246.29 & $\mathrm{O}$ & $\mathrm{CH}_{2}$ & $\mathrm{CN}$ & $\mathrm{H}$ & $\mathrm{H}$ & 81 & 93 & $>300$ \\
\hline $4 b$ & $\mathrm{C}_{13} \mathrm{H}_{12} \mathrm{~N}_{2} \mathrm{O}_{2} \mathrm{~S}$ & 260.31 & $\mathrm{O}$ & $\left(\mathrm{CH}_{2}\right)_{2}$ & $\mathrm{CN}$ & $\mathrm{H}$ & $\mathrm{H}$ & 76 & 85 & $232-233$ \\
\hline $4 c$ & $\mathrm{C}_{14} \mathrm{H}_{14} \mathrm{~N}_{2} \mathrm{O}_{2} \mathrm{~S}$ & 274.34 & $\mathrm{O}$ & $\left(\mathrm{CH}_{2}\right)_{3}$ & $\mathrm{CN}$ & $\mathrm{H}$ & $\mathrm{H}$ & 66 & 78 & $213-216$ \\
\hline $4 d$ & $\mathrm{C}_{13} \mathrm{H}_{13} \mathrm{NO}_{3} \mathrm{~S}$ & 263.32 & $\mathrm{O}$ & $\mathrm{CH}_{2}$ & $\mathrm{Ac}$ & $\mathrm{H}$ & $\mathrm{H}$ & 61 & 66 & $220-224$ \\
\hline $4 d$ & $\mathrm{C}_{13} \mathrm{H}_{13} \mathrm{NO}_{3} \mathrm{~S}$ & 263.32 & $\mathrm{~S}$ & $\mathrm{CH}_{2}$ & Ac & $\mathrm{H}$ & $\mathrm{H}$ & 67 & 77 & $220-224$ \\
\hline $4 e$ & $\mathrm{C}_{14} \mathrm{H}_{15} \mathrm{NO}_{3} \mathrm{~S}$ & 277.34 & $\mathrm{~S}$ & $\left(\mathrm{CH}_{2}\right)_{2}$ & Ac & $\mathrm{H}$ & $\mathrm{H}$ & 25 & 42 & $179-181$ \\
\hline $4 f$ & $\mathrm{C}_{13} \mathrm{H}_{13} \mathrm{NO}_{4} \mathrm{~S}$ & 279.32 & $\mathrm{O}$ & $\mathrm{CH}_{2}$ & $\mathrm{CO}_{2} \mathrm{Me}$ & $\mathrm{H}$ & $\mathrm{H}$ & 46 & 59 & $238-241$ \\
\hline $4 f$ & $\mathrm{C}_{13} \mathrm{H}_{13} \mathrm{NO}_{4} \mathrm{~S}$ & 279.32 & $\mathrm{~S}$ & $\mathrm{CH}_{2}$ & $\mathrm{CO}_{2} \mathrm{Me}$ & $\mathrm{H}$ & $\mathrm{H}$ & 68 & 73 & $238-241$ \\
\hline $4 g$ & $\mathrm{C}_{14} \mathrm{H}_{15} \mathrm{NO}_{4} \mathrm{~S}$ & 293.34 & $\mathrm{~S}$ & $\left(\mathrm{CH}_{2}\right)_{2}$ & $\mathrm{CO}_{2} \mathrm{Me}$ & $\mathrm{H}$ & $\mathrm{H}$ & 33 & 53 & $191-196$ \\
\hline $4 h$ & $\mathrm{C}_{15} \mathrm{H}_{17} \mathrm{NO}_{4} \mathrm{~S}$ & 307.37 & $\mathrm{~S}$ & $\left(\mathrm{CH}_{2}\right)_{3}$ & $\mathrm{CO}_{2} \mathrm{Me}$ & $\mathrm{H}$ & $\mathrm{H}$ & 68 & 64 & $215-219$ \\
\hline $4 i$ & $\mathrm{C}_{13} \mathrm{H}_{12} \mathrm{~N}_{2} \mathrm{O}_{2} \mathrm{~S}$ & 260.31 & $\mathrm{O}$ & $\mathrm{CH}_{2}$ & $\mathrm{CN}$ & $\mathrm{H}$ & $\mathrm{Me}$ & 88 & 93 & $265-268$ \\
\hline $4 j$ & $\mathrm{C}_{12} \mathrm{H}_{9} \mathrm{FN}_{2} \mathrm{O}_{2} \mathrm{~S}$ & 264.28 & $\mathrm{O}$ & $\mathrm{CH}_{2}$ & $\mathrm{CN}$ & $\mathrm{H}$ & $\mathrm{F}$ & 67 & 72 & $273-275$ \\
\hline $4 k$ & $\mathrm{C}_{12} \mathrm{H}_{9} \mathrm{ClN}_{2} \mathrm{O}_{2} \mathrm{~S}$ & 280.73 & $\mathrm{O}$ & $\mathrm{CH}_{2}$ & $\mathrm{CN}$ & $\mathrm{H}$ & $\mathrm{Cl}$ & 83 & 87 & $288-292$ \\
\hline 41 & $\mathrm{C}_{13} \mathrm{H}_{12} \mathrm{~N}_{2} \mathrm{O}_{2} \mathrm{~S}$ & 260.31 & $\mathrm{O}$ & $\mathrm{CH}_{2}$ & $\mathrm{CN}$ & $\mathrm{Me}$ & $\mathrm{H}$ & 95 & 97 & $>300$ \\
\hline $4 m$ & $\mathrm{C}_{12} \mathrm{H}_{9} \mathrm{FN}_{2} \mathrm{O}_{2} \mathrm{~S}$ & 264.28 & $\mathrm{O}$ & $\mathrm{CH}_{2}$ & $\mathrm{CN}$ & $\mathrm{F}$ & $\mathrm{H}$ & 88 & 93 & $>300$ \\
\hline $4 n$ & $\mathrm{C}_{12} \mathrm{H}_{9} \mathrm{ClN}_{2} \mathrm{O}_{2} \mathrm{~S}$ & 280.73 & $\mathrm{O}$ & $\mathrm{CH}_{2}$ & $\mathrm{CN}$ & $\mathrm{Cl}$ & $\mathrm{H}$ & 88 & 91 & $>300$ \\
\hline 40 & $\mathrm{C}_{14} \mathrm{H}_{14} \mathrm{~N}_{2} \mathrm{O}_{2} \mathrm{~S}$ & 274.34 & $\mathrm{O}$ & $\left(\mathrm{CH}_{2}\right)_{2}$ & $\mathrm{CN}$ & $\mathrm{H}$ & $\mathrm{Me}$ & 77 & 84 & $181-184$ \\
\hline $4 p$ & $\mathrm{C}_{13} \mathrm{H}_{11} \mathrm{FN}_{2} \mathrm{O}_{2} \mathrm{~S}$ & 278.30 & $\mathrm{O}$ & $\left(\mathrm{CH}_{2}\right)_{2}$ & $\mathrm{CN}$ & $\mathrm{H}$ & $\mathrm{F}$ & 67 & 78 & $198-201$ \\
\hline $4 q$ & $\mathrm{C}_{13} \mathrm{H}_{11} \mathrm{ClN}_{2} \mathrm{O}_{2} \mathrm{~S}$ & 294.75 & $\mathrm{O}$ & $\left(\mathrm{CH}_{2}\right)_{2}$ & $\mathrm{CN}$ & $\mathrm{H}$ & $\mathrm{Cl}$ & 68 & 80 & $215-218$ \\
\hline $4 r$ & $\mathrm{C}_{14} \mathrm{H}_{14} \mathrm{~N}_{2} \mathrm{O}_{2} \mathrm{~S}$ & 274.34 & $\mathrm{O}$ & $\left(\mathrm{CH}_{2}\right)_{2}$ & $\mathrm{CN}$ & $\mathrm{Me}$ & $\mathrm{H}$ & 90 & 97 & $210-212$ \\
\hline $4 s$ & $\mathrm{C}_{13} \mathrm{H}_{11} \mathrm{FN}_{2} \mathrm{O}_{2} \mathrm{~S}$ & 278.30 & $\mathrm{O}$ & $\left(\mathrm{CH}_{2}\right)_{2}$ & $\mathrm{CN}$ & $\mathrm{F}$ & $\mathrm{H}$ & 78 & 85 & $195-198$ \\
\hline $4 t$ & $\mathrm{C}_{13} \mathrm{H}_{11} \mathrm{ClN}_{2} \mathrm{O}_{2} \mathrm{~S}$ & 294.75 & $\mathrm{O}$ & $\left(\mathrm{CH}_{2}\right)_{2}$ & $\mathrm{CN}$ & $\mathrm{Cl}$ & $\mathrm{H}$ & 89 & 94 & $188-190$ \\
\hline $4 u$ & $\mathrm{C}_{15} \mathrm{H}_{16} \mathrm{~N}_{2} \mathrm{O}_{2} \mathrm{~S}$ & 288.37 & $\mathrm{O}$ & $\left(\mathrm{CH}_{2}\right)_{3}$ & $\mathrm{CN}$ & $\mathrm{H}$ & $\mathrm{Me}$ & 69 & 72 & $160-164$ \\
\hline $4 v$ & $\mathrm{C}_{14} \mathrm{H}_{13} \mathrm{FN}_{2} \mathrm{O}_{2} \mathrm{~S}$ & 292.33 & $\mathrm{O}$ & $\left(\mathrm{CH}_{2}\right)_{3}$ & $\mathrm{CN}$ & $\mathrm{H}$ & $\mathrm{F}$ & 66 & 61 & $195-198$ \\
\hline $4 w$ & $\mathrm{C}_{14} \mathrm{H}_{13} \mathrm{ClN}_{2} \mathrm{O}_{2} \mathrm{~S}$ & 308.78 & $\mathrm{O}$ & $\left(\mathrm{CH}_{2}\right)_{3}$ & $\mathrm{CN}$ & $\mathrm{H}$ & $\mathrm{Cl}$ & 46 & 48 & $205-206$ \\
\hline $4 x$ & $\mathrm{C}_{15} \mathrm{H}_{16} \mathrm{~N}_{2} \mathrm{O}_{2} \mathrm{~S}$ & 288.37 & $\mathrm{O}$ & $\left(\mathrm{CH}_{2}\right)_{3}$ & $\mathrm{CN}$ & $\mathrm{Me}$ & $\mathrm{H}$ & 73 & 89 & $191-195$ \\
\hline $4 y$ & $\mathrm{C}_{14} \mathrm{H}_{13} \mathrm{FN}_{2} \mathrm{O}_{2} \mathrm{~S}$ & 292.33 & $\mathrm{O}$ & $\left(\mathrm{CH}_{2}\right)_{3}$ & $\mathrm{CN}$ & $\mathrm{F}$ & $\mathrm{H}$ & - & 73 & $163-165$ \\
\hline $4 z$ & $\mathrm{C}_{14} \mathrm{H}_{13} \mathrm{ClN}_{2} \mathrm{O}_{2} \mathrm{~S}$ & 308.78 & $\mathrm{O}$ & $\left(\mathrm{CH}_{2}\right)_{3}$ & $\mathrm{CN}$ & $\mathrm{Cl}$ & $\mathrm{H}$ & - & 53 & $215-217$ \\
\hline
\end{tabular}

recorded on Varian Mercury $(200 \mathrm{MHz})$ spectrometer in DMSO- $d_{6}$ using TMS as an internal standard (chemical shifts are reported in ppm). ${ }^{13} \mathrm{C}$ NMR-spectra were recorded on Bruker DRX-300 (75 MHz) spectrometer in DMSO- $d_{6}$ using TMS as an internal standard (chemical shifts are reported in ppm).

Starting (2-halogenophenyl)sulfones 1a-j [30-32], Oand S-methyl 2a-f [33,34] have been obtained as commercial substances similarly to the previously reported methods.
[(2-Fluorophenyl)sulfonyl](pyrrolidin-2-ylidene) acetonitriles and their homologues 3a-c, i-z; typical procedure.

To the solution of [(2-fluorophenyl)sulfonyl]acetonetrile 1a,d-i $(10 \mathrm{mmol})$ in DMF $(4 \mathrm{~mL})$ the correspondent O-methyllactim $2 \mathrm{a}-\mathrm{c}(13 \mathrm{mmol})$ had been added and the mixture was additionally heated for 4 to 8 hours (monitored by $\mathrm{TLC}$, eluent $-\mathrm{CHCl}_{3}$ ) at $90^{\circ} \mathrm{C}$. The reaction mixture was diluted with 2-propanol after cooling. The precipitate formed was filtered and used for further trans- 
formation without any additional purification. The compounds were isolated as the mixture of $E$ and $Z$ isomers. The compounds 3b, c, j, k, m, n, p, q, s, t, v, w, x contained a great amount of the correspondent cyclized products $4 \mathrm{~b}, \mathrm{c}, \mathrm{j}, \mathrm{k}, \mathrm{m}, \mathrm{n}, \mathrm{p}, \mathrm{q}, \mathrm{s}, \mathrm{t}, \mathrm{v}, \mathrm{w}, \mathrm{x}$ as inseparable mixtures, and their analytical samples were not isolated. The compounds $3 y, z$ were not isolated but only the products of their further cyclization $4 \mathrm{y}, \mathrm{z}$.

1) $(2 E, Z)-[(2$-fluorophenyl)sulfonyl](pyrrolidin-2ylidene)acetonitrile (3a)

Yield: $8.2 \mathrm{mmol}(82 \%)$; cream coloured solid.

LC/MS: $m / z[\mathrm{M}+\mathrm{H}]^{+}$calcd for $\mathrm{C}_{12} \mathrm{H}_{11} \mathrm{FN}_{2} \mathrm{O}_{2} \mathrm{~S}: 266.29$; found: 266.3 .

2) (2E,Z)-[(2-fluoro-5-methylphenyl)sulfonyl](pyrrolidin-2-ylidene)acetonitrile (3i)

Yield: $9.2 \mathrm{mmol}(92 \%)$; cream coloured solid.

LC/MS: $m / z[\mathrm{M}+\mathrm{H}]^{+}$calcd for $\mathrm{C}_{13} \mathrm{H}_{13} \mathrm{FN}_{2} \mathrm{O}_{2} \mathrm{~S}: 280.32$; found: 280.4 .

3) $(2 E, Z)-[(2-f l u o r o-4-m e t h y l p h e n y l) s u l f o n y l]$ (pyrrolidin-2-ylidene)acetonitrile (31)

Yield: $9.6 \mathrm{mmol}(96 \%)$; cream coloured solid.

LC/MS: $m / z[\mathrm{M}+\mathrm{H}]^{+}$calcd for $\mathrm{C}_{13} \mathrm{H}_{13} \mathrm{FN}_{2} \mathrm{O}_{2} \mathrm{~S}$ : 280.32; found: 280.4 .

4) $(2 E, Z)-[(2-f l u o r o-5-m e t h y l p h e n y l) s u l f o n y l]($ piperidin-2-ylidene)acetonitrile (3o)

Yield: $8.9 \mathrm{mmol}(89 \%)$; cream coloured solid.

LC/MS: $m / z[\mathrm{M}+\mathrm{H}]^{+}$calcd for $\mathrm{C}_{14} \mathrm{H}_{15} \mathrm{FN}_{2} \mathrm{O}_{2} \mathrm{~S}$ : 294.35; found: 294.2 .

5) $(2 E, Z)-[(2-$ fluoro-4-methylphenyl)sulfonyl $]$ (piperidin-2-ylidene)acetonitrile (3r)

Yield: $9.4 \mathrm{mmol}(94 \%)$; cream coloured solid.

LC/MS: $m / z[\mathrm{M}+\mathrm{H}]^{+}$calcd for $\mathrm{C}_{14} \mathrm{H}_{15} \mathrm{FN}_{2} \mathrm{O}_{2} \mathrm{~S}: 294.35$; found: 294.2 .

6) (2E,Z)-azepan-2-ylidene[(2-fluoro-5-methylphenyl)sulfonyl]acetonitrile $(3 \mathrm{u})$

Yield: $7.6 \mathrm{mmol}$ (76\%); cream coloured solid.

LC/MS: $m / z[\mathrm{M}+\mathrm{H}]^{+}$calcd for $\mathrm{C}_{15} \mathrm{H}_{17} \mathrm{FN}_{2} \mathrm{O}_{2} \mathrm{~S}: 308.37$; found: 308.4 .

(1E)-1-[(2-Fluorophenyl)sulfonyl]-1-(pyrrolidin-2ylidene)acetone (3d) and (1e)-1-[(2-fluorophenyl) sulfonyl]-1-(piperidin-2-ylidene)acetone (3e); typical procedure.

The mixture of 1-[(2-fluorophenyl)sulfonyl]acetone $1 \mathrm{~b}$ (10 mmol) with the corresponding S-methyllactim $2 \mathrm{~d}$,e ( $15 \mathrm{mmol}$ ) was heated at $90^{\circ} \mathrm{C}$ for 6 to 8 hours (monitored by $\mathrm{TLC}$, eluent $-\mathrm{CHCl}_{3}$ ). The reaction mixture was diluted with 2-propanol after cooling. The precipitate formed was filtered and used for further transformation without any additional purification. The interaction of the starting ketone $1 \mathrm{~b}$ with 7-(methylthio)-3,4,5,6-tetrahydro- $2 \mathrm{H}$-azepine $2 \mathrm{f}$ resulted 2 -methyl-1,4-benzoxathiine4,4-dioxide 5.

1) (1E)-1-[(2-fluorophenyl)sulfonyl]-1-(pyrrolidin-2ylidene)acetone (3d)
Yield: $6.8 \mathrm{mmol}$ (68\%); cream coloured solid (This compound has been also obtained according to the similar procedure by the interaction between 1-[(2-fluorophenyl)sulfonyl]acetone $1 \mathrm{~b}$ and 5-methoxy-3,4-dihydro2H-pyrrole 2a. Yield: $7.3 \mathrm{mmol}(73 \%))$.

IR (KBr): 3203, 2980, 2953, 2891, 1595, 1667, 1473, 1454, 1411, 1261, 1235, 1145, 1122, 1076, 994, 820, 761, $690,595,573,529 \mathrm{~cm}^{-1}$.

${ }^{1} \mathrm{H}$ NMR (200 MHz DMSO- $\left.d_{6}\right): \delta=1.81-1.96(\mathrm{~m}, 2$ $\left.\mathrm{H}, \mathrm{CH}_{2}\right), 2.15\left(\mathrm{~s}, 3 \mathrm{H}, \mathrm{COCH}_{3}\right), 3.09(\mathrm{t}, \mathrm{J}=8.0 \mathrm{~Hz}, 2 \mathrm{H}$, $\left.\mathrm{CH}_{2}\right), 3.58$ (t, J = 7.2 Hz, $\left.2 \mathrm{H}, \mathrm{CH}_{2}\right), 7.35-7.44(\mathrm{~m}, 2 \mathrm{H}$, $\mathrm{CH}), 7.63$ - $7.74(\mathrm{~m}, 1 \mathrm{H}, \mathrm{CH}), 7.85$ - 7.94 (m, 1 H, CH), 11.50 (br s, $1 \mathrm{H}, \mathrm{NH})$.

LC/MS: $m / z[\mathrm{M}+\mathrm{H}]^{+}$calcd for $\mathrm{C}_{13} \mathrm{H}_{14} \mathrm{FNO}_{3} \mathrm{~S}$ : 283.32; found: 283.4 .

2) (1E)-1-[(2-fluorophenyl)sulfonyl]-1-(piperidin-2ylidene)acetone (3e)

Yield: $3.1 \mathrm{mmol}$ (31\%); cream coloured solid.

IR (KBr): 2975, 2940, 2873, 1606, 1580, 1466, 1444, 1304, 1216, 1152, 1111, 1018, 972, 867, 820, 765, 730, $688,622,598,573,531,456 \mathrm{~cm}^{-1}$.

${ }^{1} \mathrm{H}$ NMR (200 MHz DMSO- $\left.d_{6}\right): \delta=1.49-1.71(\mathrm{~m}, 4$ $\left.\mathrm{H}, \mathrm{CH}_{2}\right), 2.23\left(\mathrm{~s}, 3 \mathrm{H}, \mathrm{COCH}_{3}\right), 2.73(\mathrm{t}, \mathrm{J}=6.1 \mathrm{~Hz}, 2 \mathrm{H}$, $\left.\mathrm{CH}_{2}\right), 3.42\left(\mathrm{t}, \mathrm{J}=5.9 \mathrm{~Hz}, 2 \mathrm{H}, \mathrm{CH}_{2}\right), 7.35-7.46(\mathrm{~m}, 2 \mathrm{H}$, $\mathrm{CH}), 7.63$ - $7.74(\mathrm{~m}, 1 \mathrm{H}, \mathrm{CH}), 7.83$ - 7.92 (m, 1 H, CH), 13.00 (br s, $1 \mathrm{H}, \mathrm{NH}$ ).

LC/MS: $m / z[\mathrm{M}+\mathrm{H}]^{+}$calcd for $\mathrm{C}_{14} \mathrm{H}_{16} \mathrm{FNO}_{3} \mathrm{~S}: 297.35$; found: 297.4 .

Methyl (2E,Z)-[(2-fluorophenyl)sulfonyl](pyrrolidin-2-ylidene)acetate and its homologues $3 \mathrm{f}-\mathrm{h}$; typical procedure.

The mixture of methyl 1-[(2-fluorophenyl)sulfonyl] acetate $1 \mathrm{c}(10 \mathrm{mmol})$ with the corresponding S-methyllactim $2 \mathrm{~d}-\mathrm{f}(15 \mathrm{mmol})$ was heated at $100^{\circ} \mathrm{C}$ for 10 to 20 hours (monitored by TLC, eluent $-\mathrm{CHCl}_{3}$ ). The reaction mixture was diluted with 2-propanol after cooling. The precipitate formed was filtered and used for further transformation without any additional purification. The compounds were isolated as the mixture of $E$ and $Z$ isomers.

1) Methyl $(2 E, Z)-[(2-$ fluorophenyl)sulfonyl] (pyrrolidin-2-ylidene)acetate $(3 \mathrm{f})$

Yield: $8.5 \mathrm{mmol}(85 \%)$; cream coloured solid. (This compound has been also obtained according to the similar procedure by the interaction between methyl 1-[(2fluorophenyl)sulfonyl]acetate $1 \mathrm{c}$ and 5-methoxy-3,4-dihydro-2H-pyrrole 2a. Yield: $5.7 \mathrm{mmol}(57 \%)$ ).

LC/MS: $m / z[\mathrm{M}+\mathrm{H}]^{+}$calcd for $\mathrm{C}_{13} \mathrm{H}_{14} \mathrm{FNO}_{4} \mathrm{~S}$ : 299.32; found: 299.4 .

2) Methyl $(2 E, Z)-[(2-f l u o r o p h e n y l) s u l f o n y l](p i p e r i d i n-$ 2-ylidene)acetate $(3 \mathrm{~g})$

Yield: $4.2 \mathrm{mmol}$ (42\%); cream coloured solid.

LC/MS: $m / z[\mathrm{M}+\mathrm{H}]^{+}$calcd for $\mathrm{C}_{14} \mathrm{H}_{16} \mathrm{FNO}_{4} \mathrm{~S}$ : 313.36 ; found: 313.4 . 
3) Methyl (2E,Z)-azepan-2-ylidene[(2-fluorophenyl)sulfonyl]acetate ( $3 \mathrm{~h})$

Yield: $4.8 \mathrm{mmol}$ (48\%); cream coloured solid.

LC/MS: $m / z[\mathrm{M}+\mathrm{H}]^{+}$calcd for $\mathrm{C}_{15} \mathrm{H}_{18} \mathrm{FNO}_{4} \mathrm{~S}: 327.37$; found: 327.4 .

General procedure for synthesis of compounds 4a-x (cyclization of compounds 3 ).

The mixture of the correspondent compound 3 (10 $\mathrm{mmol})$, 1,4-dioxane $(6 \mathrm{ml})$ and $\mathrm{DBU}(10 \mathrm{mmol})$ was heated at $60^{\circ} \mathrm{C}$ for 2 to 4 hours (for $\mathrm{R}^{1}=\mathrm{CN} 50^{\circ} \mathrm{C}, 1-2$ hours) (monitored by TLC, eluent-2-propanole- $\mathrm{CHCl}_{3}$, $1: 30)$. In the case when the compound 3 contained the admixture of cyclization product 4 , the ratio of 3 and 4 in the mixture was calculated on the basis of integral intensity of specific peaks in ${ }^{1} \mathrm{H}$ NMR-spectra of mixture. The reaction mixture was diluted with 2-propanol after cooling. The precipitate formed was filtered and crystallized from 2-propanol-DMF mixture. The compounds $4 \mathrm{y}, \mathrm{z}$ were formed at the first stage of reaction between 7-(methylthio)-3,4,5,6-tetrahydro- $2 \mathrm{H}$-azepine $2 \mathrm{f}$ and corresponding sulfones $1 \mathrm{~h}$, i.

General procedure for synthesis of compounds $4 \mathrm{a}-\mathrm{z}$ ("one-pot" method).

To the solution of $1(10 \mathrm{mmol})$ in DMF $(4 \mathrm{~mL})$ the correspondent lactim $2(13 \mathrm{mmol})$ had been added and the mixture was additionally heated for 4 to 8 hours (monitored by TLC, eluent $-\mathrm{CHCl}_{3}$ ) at $90^{\circ} \mathrm{C}$. After the reaction mixture was cooled to room temperature, 1,4dioxane and equimolar amount of DBU were added and the reaction mixture was heated additionally at $60^{\circ} \mathrm{C}$ for 2 - 4 hours. The precipitate formed after dilution of reaction mixture with 2-propanol, was filtered and crystallized from DMF-2-propanol mixture.

1) 2,3-Dihydro- $1 H$-pyrrolo[2,1-c] $[1,4]$ benzothiazine4-carbonitrile 5,5-dioxide (4a)

Yield: $8.1 \mathrm{mmol}$ (81\%), $9.3 \mathrm{mmol}$ (93\%) ("one-pot" method); cream coloured solid.

IR (KBr): 2206, 1609, 1591, 1554, 1481, 1419, 1279 , $1152,1132,1074,769,607,575 \mathrm{~cm}^{-1}$.

${ }^{1} \mathrm{H}$ NMR (200 MHz DMSO- $\left.d_{6}\right): \delta=2.16-2.31(\mathrm{~m}, 2$ $\left.\mathrm{H}, \mathrm{CH}_{2}\right), 3.23\left(\mathrm{t}, \mathrm{J}=7.8 \mathrm{~Hz}, 2 \mathrm{H}, \mathrm{CH}_{2}\right), 4.26(\mathrm{t}, \mathrm{J}=7.2$ $\left.\mathrm{Hz}, 2 \mathrm{H}, \mathrm{CH}_{2}\right), 7.51-7.59(\mathrm{~m}, 2 \mathrm{H}, \mathrm{CH}), 7.82(\mathrm{~m}, 1 \mathrm{H}$, $\mathrm{CH}), 8.02(\mathrm{~d}, \mathrm{~J}=8.1 \mathrm{~Hz}, 1 \mathrm{H}, \mathrm{CH})$.

${ }^{13} \mathrm{C}$ NMR (75 MHz DMSO- $d_{6}$ ): $\delta=19.59,33.74$, $53.40,81.92,112.63,117.91,122.61,125.13,126.00$, 133.73, 134.25, 161.32.

LC/MS: $m / z[\mathrm{M}+\mathrm{H}]^{+}$calcd for $\mathrm{C}_{12} \mathrm{H}_{10} \mathrm{~N}_{2} \mathrm{O}_{2} \mathrm{~S}: 246.29$; found: 246.1 .

2) $7,8,9,10$-Tetrahydropyrido[2,1-c] $[1,4]$ benzothiazine-6-carbonitrile 5,5-dioxide (4b)

Yield: $7.6 \mathrm{mmol}(76 \%), 8.5 \mathrm{mmol}(85 \%)$ ("one-pot" method); cream coloured solid.

IR (KBr): 2957, 2881, 2203, 1581, 1533, 1469, 1350, $1278,1139,1073,767,609,572 \mathrm{~cm}^{-1}$.
${ }^{1} \mathrm{H}$ NMR (200 MHz DMSO- $\left.d_{6}\right): \delta=1.73-2.01(\mathrm{~m}, 4$ $\left.\mathrm{H}, \mathrm{CH}_{2}\right), 3.02\left(\mathrm{t}, \mathrm{J}=6.7 \mathrm{~Hz}, 2 \mathrm{H}, \mathrm{CH}_{2}\right), 4.09(\mathrm{t}, \mathrm{J}=5.9$ $\left.\mathrm{Hz}, 2 \mathrm{H}, \mathrm{CH}_{2}\right), 7.55-7.66(\mathrm{~m}, 1 \mathrm{H}, \mathrm{CH}), 7.78-7.85(\mathrm{~m}$, $2 \mathrm{H}, \mathrm{CH}), 8.01(\mathrm{~d}, \mathrm{~J}=8.0 \mathrm{~Hz}, 1 \mathrm{H}, \mathrm{CH})$.

${ }^{13} \mathrm{C}$ NMR (75 MHz DMSO- $\left.d_{6}\right): \delta=17.40,21.56$, $29.84,48.07,84.63,112.65,117.98,121.78,126.05$, $126.36,133.50,138.13,159.89$.

LC/MS: $m / z[\mathrm{M}+\mathrm{H}]^{+}$calcd for $\mathrm{C}_{13} \mathrm{H}_{12} \mathrm{~N}_{2} \mathrm{O}_{2} \mathrm{~S}: 260.31$; found: 260.1 .

3) $8,9,10,11$-Tetrahydro- $7 H$-azepino[2,1-c] [1,4]benzothiazine-6-carbonitrile 5,5-dioxide (4c)

Yield: $6.6 \mathrm{mmol}(66 \%), 7.8 \mathrm{mmol}$ (78\%) ("one-pot" method); cream coloured solid.

IR (KBr): 2940, 2862, 2204, 1580, 1535, 1457, 1415, $1299,1215,1167,1073,777,717,636,588 \mathrm{~cm}^{-1}$.

${ }^{1} \mathrm{H}$ NMR (200 MHz DMSO- $d_{6}$ ): $\delta=1.72$ (br s, $4 \mathrm{H}$, $\mathrm{CH}_{2}$ ), 1.83 (br s, $2 \mathrm{H}, \mathrm{CH}_{2}$ ), 3.18 (br s, $2 \mathrm{H}, \mathrm{CH}_{2}$ ), 4.31 $4.35\left(\mathrm{~m}, 2 \mathrm{H}, \mathrm{CH}_{2}\right), 7.54-7.62(\mathrm{~m}, 1 \mathrm{H}, \mathrm{CH}), 7.75-7.88$ $(\mathrm{m}, 2 \mathrm{H}, \mathrm{CH}), 7.98(\mathrm{~d}, \mathrm{~J}=8.1 \mathrm{~Hz}, 1 \mathrm{H}, \mathrm{CH})$.

${ }^{13} \mathrm{C}$ NMR (75 MHz DMSO- $\left.d_{6}\right): \delta=24.97,26.14$, $27.44,34.37,50.84,86.61,112.73,118.29,121.69$, 125.76, 126.02, 133.72, 138.62, 164.44.

LC/MS: $m / z[\mathrm{M}+\mathrm{H}]^{+}$calcd for $\mathrm{C}_{14} \mathrm{H}_{14} \mathrm{~N}_{2} \mathrm{O}_{2} \mathrm{~S}: 274.34$; found: 274.2 .

4) 1-(5,5-Dioxido-2,3-dihydro-1 $H$-pyrrolo[2,1-c] $[1,4]$ benzothiazin-4-yl)ethanone (4d)

Yield: $6.1 \mathrm{mmol}$ (61\%), $7.7 \mathrm{mmol}$ (77\%) ("one-pot" method); cream coloured solid.

IR (KBr): 3077, 2954, 2885, 1659, 1587, 1525, 1472 , $1360,1338,1283,1261,1239,1201,1136,1100,995$, $954,933,759,593,558 \mathrm{~cm}^{-1}$.

${ }^{1} \mathrm{H}$ NMR (200 MHz DMSO- $\left.d_{6}\right): \delta=2.07-2.22(\mathrm{~m}, 2$ $\left.\mathrm{H}, \mathrm{CH}_{2}\right), 2.52\left(\mathrm{~s}, 3 \mathrm{H}, \mathrm{COCH}_{3}\right), 3.34(\mathrm{t}, \mathrm{J}=7.8 \mathrm{~Hz}, 2 \mathrm{H}$, $\left.\mathrm{CH}_{2}\right), 4.15\left(\mathrm{t}, \mathrm{J}=7.3 \mathrm{~Hz}, 2 \mathrm{H}, \mathrm{CH}_{2}\right), 7.45-7.53(\mathrm{~m}, 2 \mathrm{H}$, $\mathrm{CH}), 7.60(\mathrm{t}, \mathrm{J}=7.9 \mathrm{~Hz}, 1 \mathrm{H}, \mathrm{CH}), 8.01(\mathrm{~d}, \mathrm{~J}=7.8 \mathrm{~Hz}, 1$ $\mathrm{H}, \mathrm{CH})$.

${ }^{13} \mathrm{C}$ NMR (75 MHz DMSO- $\left.d_{6}\right): \delta=20.22,30.78$, $35.10,51.74,110.13,117.29,122.88,125.26,127.10$, 133.22, 134.48, 159.63, 189.73 .

LC/MS: $m / z[\mathrm{M}+\mathrm{H}]^{+}$calcd for $\mathrm{C}_{13} \mathrm{H}_{13} \mathrm{NO}_{3} \mathrm{~S}: 263.32$; found: 263.0 .

5) 1-(5,5-Dioxido-7,8,9,10-tetrahydropyrido[2,1-c][1,4]benzothiazin-6-yl)ethanone (4e)

Yield: $2.5 \mathrm{mmol}$ (25\%), $4.2 \mathrm{mmol}$ (42\%) ("one-pot" method); cream coloured solid.

IR (KBr): 2968, 1665, 1582, 1520, 1467, 1404, 1356, $1274,1155,1137,1110,1004,911,767,625,595,564$, $511 \mathrm{~cm}^{-1}$.

${ }^{1} \mathrm{H}$ NMR (200 MHz DMSO- $\left.d_{6}\right): \delta=1.70-1.89$ (m, 4 $\left.\mathrm{H}, \mathrm{CH}_{2}\right), 2.52\left(\mathrm{~s}, 3 \mathrm{H}, \mathrm{COCH}_{3}\right), 3.04(\mathrm{t}, \mathrm{J}=7.0 \mathrm{~Hz}, 2 \mathrm{H}$, $\left.\mathrm{CH}_{2}\right), 4.10\left(\mathrm{t}, \mathrm{J}=5.7 \mathrm{~Hz}, 2 \mathrm{H}, \mathrm{CH}_{2}\right), 7.45-7.53(\mathrm{~m}, 1 \mathrm{H}$, $\mathrm{CH}), 7.70-7.80(\mathrm{~m}, 2 \mathrm{H}, \mathrm{CH}), 7.94(\mathrm{~d}, \mathrm{~J}=7.5 \mathrm{~Hz}, 1 \mathrm{H}$, $\mathrm{CH})$.

${ }^{13} \mathrm{C}$ NMR (75 MHz DMSO- $\left.d_{6}\right): \delta=17.35,21.23$, 
$26.33,32.15,46.15,113.10,117.76,121.72,125.11$, 126.91, 132.86, 138.97, 158.53, 191.56 .

LC/MS: $m / z[\mathrm{M}+\mathrm{H}]^{+}$calcd for $\mathrm{C}_{14} \mathrm{H}_{15} \mathrm{NO}_{3} \mathrm{~S}: 277.34$; found: 277.1 .

6) Methyl 2,3-dihydro-1H-pyrrolo[2,1-c][1,4]benzothiazine-4-carboxylate 5,5-dioxide (4f)

Yield: $6.8 \mathrm{mmol}$ (68\%), $7.3 \mathrm{mmol}$ (73\%) ("one-pot" method); cream coloured solid.

IR (KBr): 3087, 3017, 2954, 1696, 1590, 1542, 1482, $1282,1242,1113,765,595,555,502 \mathrm{~cm}^{-1}$.

${ }^{1} \mathrm{H}$ NMR (200 MHz DMSO- $\left.d_{6}\right): \delta=2.09-2.24$ (m, 2 $\left.\mathrm{H}, \mathrm{CH}_{2}\right), 3.36\left(\mathrm{t}, \mathrm{J}=7.8,2 \mathrm{H}, \mathrm{CH}_{2}\right), 3.75(\mathrm{~s}, 3 \mathrm{H}$, $\left.\mathrm{COOCH}_{3}\right), 4.16\left(\mathrm{t}, \mathrm{J}=7.3 \mathrm{~Hz}, 2 \mathrm{H}, \mathrm{CH}_{2}\right), 7.42$ - $7.49(\mathrm{~m}$, $2 \mathrm{H}, \mathrm{CH}), 7.73(\mathrm{~m}, 1 \mathrm{H}, \mathrm{CH}), 7.95(\mathrm{~d}, \mathrm{~J}=7.4 \mathrm{~Hz}, 1 \mathrm{H}$, $\mathrm{CH})$.

${ }^{13} \mathrm{C}$ NMR (75 MHz DMSO- $\left.d_{6}\right): \delta=20.02,34.78$, $51.72,52.03,100.92,117.17,123.07,125.13,127.43$, 132.96, 134.25, 159.67, 162.77 .

LC/MS: $m / z[\mathrm{M}+\mathrm{H}]^{+}$calcd for $\mathrm{C}_{13} \mathrm{H}_{13} \mathrm{NO}_{4} \mathrm{~S}: 279.32$; found: 279.1 .

7) Methyl 7,8,9,10-tetrahydropyrido[2,1-c][1,4]benzothiazine-6-carboxylate 5,5-dioxide (4g)

Yield: $3.3 \mathrm{mmol}(33 \%), 5.3 \mathrm{mmol}$ (53\%) ("one-pot" method); cream coloured solid.

IR (KBr): 3073, 2955, 2877, 1696, 1585, 1521, 1467, 1293, 1250, 1124, 976, 911, 867, 772, 584, 569, 517 $\mathrm{cm}^{-1}$.

${ }^{1} \mathrm{H}$ NMR (200 MHz DMSO- $\left.d_{6}\right): \delta=1.69-1.97(\mathrm{~m}, 4$ $\left.\mathrm{H}, \mathrm{CH}_{2}\right), 3.08\left(\mathrm{t}, \mathrm{J}=6.9,2 \mathrm{H}, \mathrm{CH}_{2}\right), 3.75(\mathrm{~s}, 3 \mathrm{H}$, $\left.\mathrm{COOCH}_{3}\right), 4.08\left(\mathrm{t}, \mathrm{J}=5.8 \mathrm{~Hz}, 2 \mathrm{H}, \mathrm{CH}_{2}\right), 7.43-7.51(\mathrm{~m}$, $1 \mathrm{H}, \mathrm{CH}), 7.68$ - 7.79 (m, $2 \mathrm{H}, \mathrm{CH}), 7.92(\mathrm{~d}, \mathrm{~J}=7.5 \mathrm{~Hz}, 1$ $\mathrm{H}, \mathrm{CH})$.

${ }^{13} \mathrm{C}$ NMR $\left(75 \mathrm{MHz}\right.$ DMSO- $\left.d_{6}\right): \delta=17.37,21.28$, 26.69, 46.19, 52.12, 104.47, 117.44, 121.94, 124.98, 127.04, 132.70, 138.91, 157.68, 162.39 .

LC/MS: $m / z[\mathrm{M}+\mathrm{H}]^{+}$calcd for $\mathrm{C}_{14} \mathrm{H}_{15} \mathrm{NO}_{4} \mathrm{~S}$ : 293.34; found: 293.2 .

8) Methyl 8,9,10,11-tetrahydro-7H-azepino[2,1-c][1,4]benzothiazine-6-carboxylate 5,5-dioxide (4h)

Yield: $6.8 \mathrm{mmol}(68 \%), 6.4 \mathrm{mmol}$ (64\%) ("one-pot" method); cream coloured solid.

IR (KBr): 3072, 2941, 2861, 1698, 1583, 1528, 1463, 1465, 1403, 1298, 1238, 1162, 1120, 986, 959, 905, 849, $774,691,589,564,546,509,464 \mathrm{~cm}^{-1}$.

${ }^{1} \mathrm{H}$ NMR (200 MHz DMSO- $\left.d_{6}\right): \delta=1.65$ (br s, $4 \mathrm{H}$, $\mathrm{CH}_{2}$ ), 1.84 (br s, $2 \mathrm{H}, \mathrm{CH}_{2}$ ), 3.06 (br s, $2 \mathrm{H}, \mathrm{CH}_{2}$ ), 3.76 (s, $\left.3 \mathrm{H}, \mathrm{COOCH}_{3}\right), 4.25-4.29\left(\mathrm{~m}, 2 \mathrm{H}, \mathrm{CH}_{2}\right), 7.44-7.51(\mathrm{~m}$, $1 \mathrm{H}, \mathrm{CH}), 7.65-7.79$ (m, $2 \mathrm{H}, \mathrm{CH}), 7.90$ (d, J = 7.7 Hz, 1 $\mathrm{H}, \mathrm{CH})$.

${ }^{13} \mathrm{C}$ NMR (75 MHz DMSO- $\left.d_{6}\right): \delta=25.27,26.31$, $26.85,31.68,49.32,52.42,105.65,117.58,121.91$, 124.81, 126.92, 132.86, 139.24, 159.65, 162.56.

LC/MS: $m / z[\mathrm{M}+\mathrm{H}]^{+}$calcd for $\mathrm{C}_{15} \mathrm{H}_{17} \mathrm{NO}_{4} \mathrm{~S}: 307.37$; found: 307.2 .
9) 7-Methyl-2,3-dihydro-1H-pyrrolo[2,1-c][1,4]benzothiazine-4-carbonitrile 5,5-dioxide (4i)

Yield: $8.8 \mathrm{mmol}$ (88\%), $9.3 \mathrm{mmol}$ (93\%) ("one-pot" method); cream coloured solid.

${ }^{1} \mathrm{H}$ NMR (200 MHz DMSO- $\left.d_{6}\right): \delta=2.14-2.30$ (m, 2 $\left.\mathrm{H}, \mathrm{CH}_{2}\right), 2.42\left(\mathrm{~s}, 3 \mathrm{H}, \mathrm{CH}_{3}\right), 3.20(\mathrm{t}, \mathrm{J}=7.9 \mathrm{~Hz}, 2 \mathrm{H}$, $\left.\mathrm{CH}_{2}\right), 4.23$ (t, J = 7.2 Hz, $\left.2 \mathrm{H}, \mathrm{CH}_{2}\right), 7.44(\mathrm{~d}, \mathrm{~J}=8.7 \mathrm{~Hz}$, $1 \mathrm{H}, \mathrm{CH}), 7.63(\mathrm{~d}, \mathrm{~J}=8.3 \mathrm{~Hz}, 1 \mathrm{H}, \mathrm{CH}), 7.82(\mathrm{~s}, 1 \mathrm{H}$, $\mathrm{CH})$.

${ }^{13} \mathrm{C}$ NMR (75 MHz DMSO- $\left.d_{6}\right): \delta=19.56,20.33$, $33.69,53.37,81.55,112.76,117.84,121.97,125.08$, 132.10, 134.54, 136.10, 160.82 .

LC/MS: $m / z[\mathrm{M}+\mathrm{H}]^{+}$calcd for $\mathrm{C}_{13} \mathrm{H}_{12} \mathrm{~N}_{2} \mathrm{O}_{2} \mathrm{~S}: 260.31$; found: 260.1

10) 7-Fluoro-2,3-dihydro-1H-pyrrolo[2,1-c][1,4]benzothiazine-4-carbonitrile 5,5-dioxide $(4 \mathrm{j})$

Yield: $6.7 \mathrm{mmol}(67 \%), 7.2 \mathrm{mmol}$ (72\%) ("one-pot" method); cream coloured solid.

${ }^{1} \mathrm{H}$ NMR (200 MHz DMSO- $\left.d_{6}\right): \delta=2.16-2.31$ (m, 2 $\mathrm{H}, \mathrm{CH}_{2}$ ), 3.22 (t, J = 7.8 Hz, $\left.2 \mathrm{H}, \mathrm{CH}_{2}\right), 4.26$ (t, J = 7.2 $\left.\mathrm{Hz}, 2 \mathrm{H}, \mathrm{CH}_{2}\right), 7.60-7.79(\mathrm{~m}, 2 \mathrm{H}, \mathrm{CH}), 7.93(\mathrm{dd}, \mathrm{J}(1)=$ $7.7 \mathrm{~Hz}, \mathrm{~J}(2)=2.6 \mathrm{~Hz}, 1 \mathrm{H}, \mathrm{CH})$.

${ }^{13} \mathrm{C}$ NMR (75 MHz DMSO- $\left.d_{6}\right): \delta=19.43,33.66$, $53.73,80.85,108.75,109.01,112.36,120.87,120.95$, $121.40,121.63,125.92,125.99,131.13,157.45,159.91$, 161.21 .

LC/MS: $m / z[\mathrm{M}+\mathrm{H}]^{+}$calcd for $\mathrm{C}_{12} \mathrm{H}_{9} \mathrm{FN}_{2} \mathrm{O}_{2} \mathrm{~S}: 264.28$; found: 264.3 .

11) 7-Chloro-2,3-dihydro-1H-pyrrolo[2,1-c][1,4]benzothiazine-4-carbonitrile 5,5-dioxide (4k)

Yield: $8.3 \mathrm{mmol}$ (83\%), $8.7 \mathrm{mmol}$ (87\%) ("one-pot' method); cream coloured solid.

${ }^{1} \mathrm{H}$ NMR (200 MHz DMSO- $\left.d_{6}\right): \delta=2.16-2.31(\mathrm{~m}, 2$ $\mathrm{H}, \mathrm{CH}_{2}$ ), 3.22 (t, J = 7.8 Hz, $\left.2 \mathrm{H}, \mathrm{CH}_{2}\right), 4.25$ (t, J = 7.3 $\mathrm{Hz}, 2 \mathrm{H}, \mathrm{CH}_{2}$ ), 7.59 (d, J = 9.2 Hz, 1 H, CH), 7.89 (dd, $\mathrm{J}(1)=9.0 \mathrm{~Hz}, \mathrm{~J}(2)=2.4 \mathrm{~Hz}, 1 \mathrm{H}, \mathrm{CH}), 8.65(\mathrm{~d}, \mathrm{~J}=2.5$ $\mathrm{Hz}, 1 \mathrm{H}, \mathrm{CH})$.

${ }^{13} \mathrm{C}$ NMR (75 MHz DMSO- $\left.d_{6}\right): \delta=19.58,33.81$, $53.74,82.18,112.28,120.42,121.91,126.21,129.89$, 133.30, 133.68, 161.61.

LC/MS: $m / z[\mathrm{M}+\mathrm{H}]^{+}$calcd for $\mathrm{C}_{12} \mathrm{H}_{9} \mathrm{ClN}_{2} \mathrm{O}_{2} \mathrm{~S}$ : 280.73; found: 280.3 .

12) 8-Methyl-2,3-dihydro-1H-pyrrolo[2,1-c][1,4]benzothiazine-4-carbonitrile 5,5-dioxide (41)

Yield: $9.5 \mathrm{mmol}$ (95\%), $9.7 \mathrm{mmol}$ (97\%) (“one-pot" method); cream coloured solid.

${ }^{1} \mathrm{H}$ NMR (200 MHz DMSO- $\left.d_{6}\right): \delta=2.15-2.30$ (m, 2 $\left.\mathrm{H}, \mathrm{CH}_{2}\right), 2.45\left(\mathrm{~s}, 3 \mathrm{H}, \mathrm{CH}_{3}\right), 3.21(\mathrm{t}, \mathrm{J}=7.8 \mathrm{~Hz}, 2 \mathrm{H}$, $\left.\mathrm{CH}_{2}\right), 4.23\left(\mathrm{t}, \mathrm{J}=7.3 \mathrm{~Hz}, 2 \mathrm{H}, \mathrm{CH}_{2}\right), 7.37(\mathrm{~d}, \mathrm{~J}=8.3 \mathrm{~Hz}$, $1 \mathrm{H}, \mathrm{CH}), 7.38$ (s, $1 \mathrm{H}, \mathrm{CH}), 7.89$ (d, J = 8.6 Hz, $1 \mathrm{H}$, $\mathrm{CH})$.

${ }^{13} \mathrm{C}$ NMR (75 MHz DMSO- $\left.d_{6}\right): \delta=19.58,21.35$, $33.70,53.33,82.05,112.71,117.70,122.54,122.73$, $126.89,134.29,144.46,161.15$. 
LC/MS: $m / z[\mathrm{M}+\mathrm{H}]^{+}$calcd for $\mathrm{C}_{13} \mathrm{H}_{12} \mathrm{~N}_{2} \mathrm{O}_{2} \mathrm{~S}: 260.31$; found: 260.1 .

13) 8-Fluoro-2,3-dihydro- $1 H$-pyrrolo[ $[2,1-c][1,4]$ benzothiazine-4-carbonitrile 5,5-dioxide $(4 \mathrm{~m})$

Yield: $8.8 \mathrm{mmol}(88 \%), 9.3 \mathrm{mmol}$ (93\%) ("one-pot" method); cream coloured solid.

${ }^{1} \mathrm{H}$ NMR (200 MHz DMSO- $\left.d_{6}\right): \delta=2.16-2.31(\mathrm{~m}, 2$ $\left.\mathrm{H}, \mathrm{CH}_{2}\right), 3.23\left(\mathrm{t}, \mathrm{J}=7.8 \mathrm{~Hz}, 2 \mathrm{H}, \mathrm{CH}_{2}\right), 4.22(\mathrm{t}, \mathrm{J}=7.2$ $\left.\mathrm{Hz}, 2 \mathrm{H}, \mathrm{CH}_{2}\right), 7.36-7.55(\mathrm{~m}, 2 \mathrm{H}, \mathrm{CH}), 8.11(\mathrm{dd}, \mathrm{J}(1)=$ $8.9 \mathrm{~Hz}, \mathrm{~J}(2)=5.8 \mathrm{~Hz}, 1 \mathrm{H}, \mathrm{CH})$.

${ }^{13} \mathrm{C}$ NMR (75 MHz DMSO- $\left.d_{6}\right): \delta=19.61,33.83$, $53.78,82.87,105.03,105.39,112.30,113.70,114.01$, $121.77,121.81,125.90,126.05,136.48,136.64,161.83$, 162.64, 165.97.

LC/MS: $m / z[\mathrm{M}+\mathrm{H}]^{+}$calcd for $\mathrm{C}_{12} \mathrm{H}_{9} \mathrm{FN}_{2} \mathrm{O}_{2} \mathrm{~S}: 264.28$; found: 264.5 .

14) 8-Chloro-2,3-dihydro-1H-pyrrolo[2,1-c][1,4]benzothiazine-4-carbonitrile 5,5-dioxide (4n)

Yield: $8.8 \mathrm{mmol}$ (88\%), $9.1 \mathrm{mmol}$ (91\%) ("one-pot" method); cream coloured solid.

${ }^{1} \mathrm{H}$ NMR (200 MHz DMSO- $\left.d_{6}\right): \delta=2.15-2.30$ (m, 2 $\left.\mathrm{H}, \mathrm{CH}_{2}\right), 3.23$ (t, J = 7.8 Hz, $\left.2 \mathrm{H}, \mathrm{CH}_{2}\right), 4.25$ (t, J = 7.3 $\left.\mathrm{Hz}, 2 \mathrm{H}, \mathrm{CH}_{2}\right), 7.60(\mathrm{~d}, \mathrm{~J}=8.6 \mathrm{~Hz}, 1 \mathrm{H}, \mathrm{CH}), 7.69(\mathrm{~s}, 1 \mathrm{H}$, $\mathrm{CH}), 8.05(\mathrm{~d}, \mathrm{~J}=8.6 \mathrm{~Hz}, 1 \mathrm{H}, \mathrm{CH})$.

${ }^{13} \mathrm{C}$ NMR (75 MHz DMSO- $d_{6}$ ): $\delta=19.51,33.72$, $53.60,82.66,112.14,117.67,123.69,124.62,125.91$, 135.49, 138.28, 161.76 .

LC/MS: $m / z[\mathrm{M}+\mathrm{H}]^{+}$calcd for $\mathrm{C}_{12} \mathrm{H}_{9} \mathrm{ClN}_{2} \mathrm{O}_{2} \mathrm{~S}$ : 280.73; found: 280.3 .

15) 3-Methyl-7,8,9,10-tetrahydropyrido[2,1-c][1,4]benzothiazine-6-carbonitrile 5,5-dioxide (4o)

Yield: $7.7 \mathrm{mmol}$ (77\%), $8.4 \mathrm{mmol}$ (84\%) ("one-pot" method); cream coloured solid.

${ }^{1} \mathrm{H}$ NMR (200 MHz DMSO- $d_{6}$ ): $\delta=1.70-1.99$ (m, 4 $\left.\mathrm{H}, \mathrm{CH}_{2}\right), 2.42\left(\mathrm{~s}, 3 \mathrm{H}, \mathrm{CH}_{3}\right), 3.00(\mathrm{t}, \mathrm{J}=6.7 \mathrm{~Hz}, 2 \mathrm{H}$, $\left.\mathrm{CH}_{2}\right), 4.05\left(\mathrm{t}, \mathrm{J}=5.8 \mathrm{~Hz}, 2 \mathrm{H}, \mathrm{CH}_{2}\right), 7.64(\mathrm{~d}, \mathrm{~J}=9.3 \mathrm{~Hz}$, $1 \mathrm{H}, \mathrm{CH}), 7.73(\mathrm{~d}, \mathrm{~J}=9.2 \mathrm{~Hz}, 1 \mathrm{H}, \mathrm{CH}), 7.81(\mathrm{~s}, 1 \mathrm{H}$, $\mathrm{CH})$.

${ }^{13} \mathrm{C}$ NMR (75 MHz DMSO- $\left.d_{6}\right): \delta=17.45,20.15$, $21.59,29.80,48.06,66.44,84.17,112.82,117.92,121.15$, 126.00, 134.32, 135.96, 136.50, 159.31.

LC/MS: $m / z[\mathrm{M}+\mathrm{H}]^{+}$calcd for $\mathrm{C}_{14} \mathrm{H}_{14} \mathrm{~N}_{2} \mathrm{O}_{2} \mathrm{~S}: 274.34$; found: 274.2 .

16) 3-Fluoro-7,8,9,10-tetrahydropyrido[2,1-c][1,4]benzothiazine-6-carbonitrile 5,5-dioxide (4p)

Yield: $6.7 \mathrm{mmol}(67 \%), 7.8 \mathrm{mmol}$ (78\%) ("one-pot" method); cream coloured solid.

${ }^{1} \mathrm{H}$ NMR (200 MHz DMSO- $d_{6}$ ): $\delta=1.72-1.99$ (m, 4 $\left.\mathrm{H}, \mathrm{CH}_{2}\right), 3.02\left(\mathrm{t}, \mathrm{J}=6.7 \mathrm{~Hz}, 2 \mathrm{H}, \mathrm{CH}_{2}\right), 4.08(\mathrm{t}, \mathrm{J}=5.7$ $\left.\mathrm{Hz}, 2 \mathrm{H}, \mathrm{CH}_{2}\right), 7.69-7.79(\mathrm{~m}, 1 \mathrm{H}, \mathrm{CH}), 7.86-7.97$ (m, $2 \mathrm{H}, \mathrm{CH})$.

${ }^{13} \mathrm{C}$ NMR (75 MHz DMSO- $\left.d_{6}\right): \delta=17.29,21.42$, $29.73,48.45,83.48,107.73,107.99,112.41,120.99$, $121.26,127.04,134.94,157.66,159.88,160.13$.
LC/MS: $m / z[\mathrm{M}+\mathrm{H}]^{+}$calcd for $\mathrm{C}_{13} \mathrm{H}_{11} \mathrm{FN}_{2} \mathrm{O}_{2} \mathrm{~S}: 278.30$; found: 278.3 .

17) 3-Chloro-7,8,9,10-tetrahydropyrido[2,1-c] $[1,4]$ benzothiazine-6-carbonitrile 5,5-dioxide (4q)

Yield: $6.8 \mathrm{mmol}(68 \%), 8.0 \mathrm{mmol}(80 \%)$ ("one-pot" method); cream coloured solid.

${ }^{1} \mathrm{H}$ NMR (200 MHz DMSO- $d_{6}$ ): $\delta=1.73-2.01$ (m, 4 $\left.\mathrm{H}, \mathrm{CH}_{2}\right), 3.02\left(\mathrm{t}, \mathrm{J}=6.6 \mathrm{~Hz}, 2 \mathrm{H}, \mathrm{CH}_{2}\right), 4.08(\mathrm{t}, \mathrm{J}=5.8$ $\left.\mathrm{Hz}, 2 \mathrm{H}, \mathrm{CH}_{2}\right), 7.88-7.89(\mathrm{~m}, 2 \mathrm{H}, \mathrm{CH}), 8.01-8.03(\mathrm{~m}$, $1 \mathrm{H}, \mathrm{CH})$.

${ }^{13} \mathrm{C}$ NMR (75 MHz DMSO- $\left.d_{6}\right): \delta=17.25,21.35$, $29.77,48.31,84.60,112.25,120.52,120.87,126.94$, $130.29,133.25,136.97,160.13$.

LC/MS: $m / z[\mathrm{M}+\mathrm{H}]^{+}$calcd for $\mathrm{C}_{13} \mathrm{H}_{11} \mathrm{ClN}_{2} \mathrm{O}_{2} \mathrm{~S}$ : 294.75; found: 294.3 .

18) 2-Methyl-7,8,9,10-tetrahydropyrido $[2,1-c][1,4]$ benzothiazine-6-carbonitrile 5,5-dioxide (4r)

Yield: $9.0 \mathrm{mmol}(90 \%), 9.7 \mathrm{mmol}$ (97\%) (“one-pot" method); cream coloured solid.

${ }^{1} \mathrm{H}$ NMR $\left(200 \mathrm{MHz}\right.$ DMSO- $\left.d_{6}\right): \delta=1.73-2.00$ (m, 4 $\left.\mathrm{H}, \mathrm{CH}_{2}\right), 2.47\left(\mathrm{~s}, 3 \mathrm{H}, \mathrm{CH}_{3}\right), 3.01(\mathrm{t}, \mathrm{J}=6.7 \mathrm{~Hz}, 2 \mathrm{H}$, $\left.\mathrm{CH}_{2}\right), 4.07\left(\mathrm{t}, \mathrm{J}=6.0 \mathrm{~Hz}, 2 \mathrm{H}, \mathrm{CH}_{2}\right), 7.41(\mathrm{~d}, \mathrm{~J}=8.3 \mathrm{~Hz}$, $1 \mathrm{H}, \mathrm{CH}), 7.68(\mathrm{~s}, 1 \mathrm{H}, \mathrm{CH}), 7.88(\mathrm{~d}, \mathrm{~J}=8.1,1 \mathrm{H}, \mathrm{CH})$.

${ }^{13} \mathrm{C}$ NMR (75 MHz DMSO- $\left.d_{6}\right): \delta=17.31,21.42$, $29.71,47.86,84.66,112.65,117.76,121.60,123.52$, 127.08, 138.04, 144.09, 159.60.

LC/MS: $m / z[\mathrm{M}+\mathrm{H}]^{+}$calcd for $\mathrm{C}_{14} \mathrm{H}_{14} \mathrm{~N}_{2} \mathrm{O}_{2} \mathrm{~S}: 274.34$; found: 274.2 .

19) 2-Fluoro-7,8,9,10-tetrahydropyrido[2,1-c] [1,4]benzothiazine-6-carbonitrile 5,5-dioxide (4s)

Yield: $7.8 \mathrm{mmol}$ (78\%), $8.5 \mathrm{mmol}$ (85\%) ("one-pot" method); cream coloured solid.

${ }^{1} \mathrm{H}$ NMR (200 MHz DMSO- $d_{6}$ ): $\delta=1.72-2.00$ (m, 4 $\left.\mathrm{H}, \mathrm{CH}_{2}\right), 3.02$ (t, J = 6.7 Hz, $\left.2 \mathrm{H}, \mathrm{CH}_{2}\right), 4.03(\mathrm{t}, \mathrm{J}=6.0$ $\left.\mathrm{Hz}, 2 \mathrm{H}, \mathrm{CH}_{2}\right), 7.42-7.52(\mathrm{~m}, 1 \mathrm{H}, \mathrm{CH}), 7.78(\mathrm{dd}, \mathrm{J}(1)=$ $12.1 \mathrm{~Hz}, \mathrm{~J}(2)=2.2 \mathrm{~Hz}, 1 \mathrm{H}, \mathrm{CH}), 8.06-8.13(\mathrm{~m}, 1 \mathrm{H}$, $\mathrm{CH})$.

${ }^{13} \mathrm{C}$ NMR $\left(75 \mathrm{MHz}\right.$ DMSO- $\left.d_{6}\right): \delta=17.22,21.28$, $29.76,48.26,85.53,105.35,105.63,112.25,113.97$, $114.21,122.47,124.88,124.99,140.14,140.25,160.26$, $162.97,165.45$.

LC/MS: $m / z[\mathrm{M}+\mathrm{H}]^{+}$calcd for $\mathrm{C}_{13} \mathrm{H}_{11} \mathrm{FN}_{2} \mathrm{O}_{2} \mathrm{~S}: 278.30$; found: 278.3 .

20) 2-Chloro-7,8,9,10-tetrahydropyrido[2,1-c][1,4] benzothiazine-6-carbonitrile 5,5-dioxide (4t)

Yield: $8.9 \mathrm{mmol}$ (89\%), $9.4 \mathrm{mmol}$ (94\%) (“one-pot" method); cream coloured solid.

${ }^{1} \mathrm{H}$ NMR (200 MHz DMSO- $\left.d_{6}\right): \delta=1.72-1.99$ (m, 4 $\left.\mathrm{H}, \mathrm{CH}_{2}\right), 3.02\left(\mathrm{t}, \mathrm{J}=6.7 \mathrm{~Hz}, 2 \mathrm{H}, \mathrm{CH}_{2}\right), 4.08(\mathrm{t}, \mathrm{J}=5.9$ $\left.\mathrm{Hz}, 2 \mathrm{H}, \mathrm{CH}_{2}\right), 7.66(\mathrm{dd}, \mathrm{J}(1)=8.6 \mathrm{~Hz}, \mathrm{~J}(2)=1.7 \mathrm{~Hz}, 1 \mathrm{H}$, $\mathrm{CH}), 7.96(\mathrm{~d}, \mathrm{~J}=1.6 \mathrm{~Hz}, 1 \mathrm{H}, \mathrm{CH}), 8.03(\mathrm{~d}, \mathrm{~J}=8.6 \mathrm{~Hz}, 1$ $\mathrm{H}, \mathrm{CH})$.

${ }^{13} \mathrm{C}$ NMR (75 MHz DMSO- $\left.d_{6}\right): \delta=17.22,21.28$, $29.80,49.22,85.38,112.20,117.98,123.74,124.50$, 
126.31, 138.24, 139.24, 160.36 .

LC/MS: $m / z[\mathrm{M}+\mathrm{H}]^{+}$calcd for $\mathrm{C}_{13} \mathrm{H}_{11} \mathrm{ClN}_{2} \mathrm{O}_{2} \mathrm{~S}$ : 294.75; found: 294.6 .

21) 3-Methyl-8,9,10,11-tetrahydro-7H-azepino[2,1-c][1,4]benzothiazine-6-carbonitrile 5,5-dioxide (4u)

Yield: $6.9 \mathrm{mmol}$ (69\%), $7.2 \mathrm{mmol}$ (72\%) ("one-pot" method); cream coloured solid.

${ }^{1} \mathrm{H}$ NMR (200 MHz DMSO- $d_{6}$ ): $\delta=1.71$ (br s, $4 \mathrm{H}$, $\mathrm{CH}_{2}$ ), 1.81 (br s, $\left.2 \mathrm{H}, \mathrm{CH}_{2}\right), 2.42\left(\mathrm{~s}, 3 \mathrm{H}, \mathrm{CH}_{3}\right), 3.16$ (br s, $\left.2 \mathrm{H}, \mathrm{CH}_{2}\right), 4.28$ - $4.32\left(\mathrm{~m}, 2 \mathrm{H}, \mathrm{CH}_{2}\right), 7.60-7.70(\mathrm{~m}, 2 \mathrm{H}$, $\mathrm{CH}), 7.78$ (s, $1 \mathrm{H}, \mathrm{CH})$.

${ }^{13} \mathrm{C}$ NMR $\left(75 \mathrm{MHz}\right.$ DMSO- $\left.d_{6}\right): \delta=20.02,24.87$, 26.01, 27.34, 34.14, 50.60, 85.91, 112.81, 118.13, 120.86, $125.47,134.43,136.07,136.30,163.94$

LC/MS: $m / z[\mathrm{M}+\mathrm{H}]^{+}$calcd for $\mathrm{C}_{15} \mathrm{H}_{16} \mathrm{~N}_{2} \mathrm{O}_{2} \mathrm{~S}: 288.37$; found: 288.1 .

22) 3-Fluoro-8,9,10,11-tetrahydro-7H-azepino[2,1-c][1,4]benzothiazine-6-carbonitrile 5,5-dioxide (4v)

Yield: $6.6 \mathrm{mmol}(66 \%), 6.1 \mathrm{mmol}$ (61\%) ("one-pot" method); cream coloured solid.

${ }^{1} \mathrm{H}$ NMR (200 MHz DMSO- $d_{6}$ ): $\delta=1.71$ (br s, $4 \mathrm{H}$, $\mathrm{CH}_{2}$ ), 1.82 (br s, $2 \mathrm{H}, \mathrm{CH}_{2}$ ), 3.18 (br s, $2 \mathrm{H}, \mathrm{CH}_{2}$ ), 4.31 $4.35\left(\mathrm{~m}, 2 \mathrm{H}, \mathrm{CH}_{2}\right), 7.68$ - $7.78(\mathrm{~m}, 1 \mathrm{H}, \mathrm{CH}), 7.82$ - 7.90 (m, $2 \mathrm{H}, \mathrm{CH})$.

${ }^{13} \mathrm{C}$ NMR $\left(75 \mathrm{MHz}\right.$ DMSO- $\left.d_{6}\right): \delta=24.72,25.90$, $27.33,34.22,51.08,85.57,107.62,107.87,112.51$, $121.23,121.45,126.61,126.68,135.36,157.45,159.92$, 164.47.

LC/MS: $m / z[\mathrm{M}+\mathrm{H}]^{+}$calcd for $\mathrm{C}_{14} \mathrm{H}_{13} \mathrm{FN}_{2} \mathrm{O}_{2} \mathrm{~S}: 292.33$; found: 292.3 .

23) 3-Chloro-8,9,10,11-tetrahydro-7H-azepino[2,1-c][1,4]benzothiazine-6-carbonitrile 5,5-dioxide (4w)

Yield: $4.6 \mathrm{mmol}$ (46\%), $4.8 \mathrm{mmol}$ (48\%) ("one-pot" method); cream coloured solid.

${ }^{1} \mathrm{H}$ NMR (200 MHz DMSO- $d_{6}$ ): $\delta=1.70$ (br s, $4 \mathrm{H}$, $\mathrm{CH}_{2}$ ), 1.77 - $1.81\left(\mathrm{~m}, 2 \mathrm{H}, \mathrm{CH}_{2}\right.$ ), 3.18 (br s, $2 \mathrm{H}, \mathrm{CH}_{2}$ ), 4.30 - $4.34\left(\mathrm{~m}, 2 \mathrm{H}, \mathrm{CH}_{2}\right), 7.78-8.01(\mathrm{~m}, 3 \mathrm{H}, \mathrm{CH})$.

${ }^{13} \mathrm{C}$ NMR (75 MHz DMSO- $\left.d_{6}\right): \delta=24.7,25.82,27.30$, $34.28,50.96,86.66,112.36,120.81,126.60,129.92$, 133.45, 137.45, 164.67.

LC/MS: $m / z[\mathrm{M}+\mathrm{H}]^{+}$calcd for $\mathrm{C}_{14} \mathrm{H}_{13} \mathrm{ClN}_{2} \mathrm{O}_{2} \mathrm{~S}$ : 308.78; found: 308.3 .

24) 2-Methyl-8,9,10,11-tetrahydro-7H-azepino[2,1-c][1,4]benzothiazine-6-carbonitrile 5,5-dioxide (4x)

Yield: $7.3 \mathrm{mmol}$ (73\%), $8.9 \mathrm{mmol}$ (89\%) ("one-pot" method); cream coloured solid.

${ }^{1} \mathrm{H}$ NMR (200 MHz DMSO- $d_{6}$ ): $\delta=1.71$ (br s, $4 \mathrm{H}$, $\mathrm{CH}_{2}$ ), 1.83 (br s, $2 \mathrm{H}, \mathrm{CH}_{2}$ ), $2.48\left(\mathrm{~s}, 3 \mathrm{H}, \mathrm{CH}_{3}\right.$ ), 3.17 (br s, $\left.2 \mathrm{H}, \mathrm{CH}_{2}\right), 4.29-4.33\left(\mathrm{~m}, 2 \mathrm{H}, \mathrm{CH}_{2}\right), 7.40$ (d, J = 8.0 Hz, $1 \mathrm{H}, \mathrm{CH}), 7.58$ (s, $1 \mathrm{H}, \mathrm{CH}), 7.86(\mathrm{~d}, \mathrm{~J}=7.9 \mathrm{~Hz}, 1 \mathrm{H}$, $\mathrm{CH})$.

${ }^{13} \mathrm{C}$ NMR (75 MHz DMSO- $\left.d_{6}\right): \delta=21.45,24.91$, 26.10, 27.35, 34.25, 50.57, 86.56, 112.73, 117.96, 121.48, $123.21,126.81,138.53,144.39,164.19$.
LC/MS: $m / z[\mathrm{M}+\mathrm{H}]^{+}$calcd for $\mathrm{C}_{15} \mathrm{H}_{16} \mathrm{~N}_{2} \mathrm{O}_{2} \mathrm{~S}$ : 288.37; found: 288.3 .

25) 2-Fluoro-8,9,10,11-tetrahydro-7H-azepino[2,1-c][1,4]benzothiazine-6-carbonitrile 5,5-dioxide (4y)

Yield: $7.3 \mathrm{mmol}$ (73\%) ("one-pot" method); cream coloured solid.

${ }^{1} \mathrm{H}$ NMR (200 MHz DMSO- $d_{6}$ ): $\delta=1.71$ (br s, $4 \mathrm{H}$, $\mathrm{CH}_{2}$ ), $1.78-1.81\left(\mathrm{~m}, 2 \mathrm{H}, \mathrm{CH}_{2}\right), 3.18$ (br s, $2 \mathrm{H}, \mathrm{CH}_{2}$ ), 4.28 - $4.32\left(\mathrm{~m}, 2 \mathrm{H}, \mathrm{CH}_{2}\right), 7.41-7.50(\mathrm{~m}, 1 \mathrm{H}, \mathrm{CH}), 7.69$ $(\mathrm{dd}, \mathrm{J}(1)=11.7 \mathrm{~Hz}, \mathrm{~J}(2)=2.2 \mathrm{~Hz}, 1 \mathrm{H}, \mathrm{CH}), 8.03-8.11$ (m, $1 \mathrm{H}, \mathrm{CH})$.

${ }^{13} \mathrm{C}$ NMR (75 MHz DMSO- $\left.d_{6}\right): \delta=24.92,25.91$, $27.39,34.43,51.03,87.65,105.59,105.96,112.46$, $113.80,114.11,122.26,124.89,125.04,140.62,140.78$, 162.89, 164.81, 166.21.

LC/MS: $m / z[\mathrm{M}+\mathrm{H}]^{+}$calcd for $\mathrm{C}_{14} \mathrm{H}_{13} \mathrm{FN}_{2} \mathrm{O}_{2} \mathrm{~S}: 292.33$; found: 292.4 .

26) 2-Chloro-8,9,10,11-tetrahydro-7H-azepino[2,1-c]$[1,4]$ benzothiazine-6-carbonitrile 5,5-dioxide (4z)

Yield: $5.3 \mathrm{mmol}$ (53\%) ("one-pot" method); cream coloured solid.

${ }^{1} \mathrm{H}$ NMR (200 MHz DMSO- $\left.d_{6}\right): \delta=1.71$ (br s, $4 \mathrm{H}$, $\left.\mathrm{CH}_{2}\right), 1.80-1.82\left(\mathrm{~m}, 2 \mathrm{H}, \mathrm{CH}_{2}\right), 3.16-3.19(\mathrm{~m}, 2 \mathrm{H}$, $\left.\mathrm{CH}_{2}\right), 4.31-4.35\left(\mathrm{~m}, 2 \mathrm{H}, \mathrm{CH}_{2}\right), 7.63(\mathrm{dd}, \mathrm{J}(1)=8.4 \mathrm{~Hz}$, $\mathrm{J}(2)=1.6 \mathrm{~Hz}, 1 \mathrm{H}, \mathrm{CH}), 7.84(\mathrm{~d}, \mathrm{~J}=1.5 \mathrm{~Hz}, 1 \mathrm{H}, \mathrm{CH})$, $8.00(\mathrm{~d}, \mathrm{~J}=8.6 \mathrm{~Hz}, 1 \mathrm{H}, \mathrm{CH})$.

${ }^{13} \mathrm{C}$ NMR (75 MHz DMSO- $\left.d_{6}\right): \delta=24.73,25.80$, $27.32,34.36,50.84,87.36,112.32,118.10,123.66$, 124.17, 126.03, 138.43, 139.65, 164.83.

LC/MS: $m / z[\mathrm{M}+\mathrm{H}]^{+}$calcd for $\mathrm{C}_{14} \mathrm{H}_{13} \mathrm{ClN}_{2} \mathrm{O}_{2} \mathrm{~S}$ : 308.78; found: 308.3 .

\section{2-Methyl-1,4-benzoxathiine 4,4-dioxide (5).}

The mixture of $10 \mathrm{mmol}$ [(2-fluorophenyl)sulfonyl] acetone $1 \mathrm{~b}, 10 \mathrm{mmol} \mathrm{DBU}$ and $2 \mathrm{~mL}$ of 1,4-dioxane were heated $80^{\circ} \mathrm{C}-90^{\circ} \mathrm{C}$ for $3-4$ hours (monitored by TLC, eluent $-\mathrm{CHCl}_{3}$ ). The reaction mixture was diluted with 2-propanol after cooling. The precipitate formed was filtered and crystallized from 2-propanol-DMF mixture.

Yield: $5.5 \mathrm{mmol}$ (55\%); cream coloured solid.

${ }^{1} \mathrm{H}$ NMR (200 MHz DMSO- $\left.d_{6}\right): \delta=2.23\left(\mathrm{~s}, 3 \mathrm{H}, \mathrm{CH}_{3}\right)$, $6.82(\mathrm{~s}, 1 \mathrm{H}, \mathrm{CH}), 7.43-7.53\left(\mathrm{~m}, 2 \mathrm{H}, \mathrm{CH}_{2}\right), 7.68$ - 7.77 (m, $1 \mathrm{H}, \mathrm{CH}), 7.93(\mathrm{~d}, \mathrm{~J}=7.9 \mathrm{~Hz}, 1 \mathrm{H}, \mathrm{CH})$.

${ }^{13} \mathrm{C}$ NMR (75 MHz DMSO- $\left.d_{6}\right): \delta=20.37,103.44$, 118.73, 122.43, 124.54, 126.09, 133.96, 149.90, 158.08.

LC/MS: $m / z[M+H]^{+}$calcd for $\mathrm{C}_{9} \mathrm{H}_{8} \mathrm{O}_{3} \mathrm{~S}:$ 196.22; found: 196.3 .

(2E,Z)-2-\{[(2-fluorophenyl)sulfonyl]methylidene\}azepane (6).

The mixture of 1-[(2-fluorophenyl)sulfonyl]acetone $1 \mathrm{~b}$ (10 mmol) with S-methyllactim $2 \mathrm{f}(15 \mathrm{mmol})$ was heated at $90^{\circ} \mathrm{C}$ for 6 to 8 hours (monitored by TLC, eluent$\mathrm{CHCl}_{3}$ ). The reaction mixture was diluted with 2-propanol after cooling. The precipitate formed was filtered 
and crystallized from 2-propanol-DMF mixture.

Yield: $2.5 \mathrm{mmol}(25 \%)$; cream coloured solid.

LC/MS: $m / z[\mathrm{M}+\mathrm{H}]^{+}$calcd for $\mathrm{C}_{9} \mathrm{H}_{8} \mathrm{O}_{3} \mathrm{~S}: 269.34$; found: 269.5 .

\section{Conclusion}

The reaction of different methylene active (2-fluorophenyl)sulfones with O- and S-methylactims has been studied. A facile one-pot method for synthesis of 2,3dihydro- $1 H$-pyrrolo[2,1-c][1,4]benzothiazines and their homologues by interaction of methylenactive (2-fluorophenyl)sulfones with homologues of either 5-methoxy3,4-dihydro-2H-pyrrole or 5-(methylthio)-3,4-dihydro$2 H$-pyrrole has been developed.

\section{REFERENCES}

[1] F. Varano, D. Catarzi, V. Colotta, G. Filacchioni, L. Cecchi, A. Galli and C. Costagli, "Synthesis of 2-Sub-stituted-6,8-dichloro-3,4-dihydro-3-oxo-2H-1,4-benzothiazi ne-1,1-dioxides and -1-Oxides as Glycine-NMDA Receptor Antagonists," Il Farmaco, Vol. 53, No. 12, 1998, pp. 752-757. doi:10.1016/S0014-827X(98)00097-4

[2] P. Rafferty, D. Calderwood, L. D. Arnold, B. Gonzalez Pascual, J. L. Ortego Matinez, M. J. Perez de Vega and I. F. Fernandez, "Benzothiazinone and Benzoxazinone Compounds," PCT Int. Appl. WO 2000075139, 2000.

[3] J. F. Blake, J. B.Fell, J. P. Fischer, R. T. Hendricks, J. E. Robinson, S. R. Spencer and P. J. Stengel, "Heterocyclic Antiviral Compounds," US Patent No. 2006040927, 2006.

[4] H. C. Hansen, T. M. Tagmose and J. B. Hansen, "Fused 1,4-Thiazine-2-carbonitrile Derivatives, Their Preparation and Use," PCT Int. Appl. WO 2000055147, 2000.

[5] T. Yamamoto, I. Watanabe, K. Harada and S. Ikeda, "Benzo[1,4]thiazine Derivatives and Drugs Comprising the Same," PCT Int. Appl. WO 9813357, 1998.

[6] I. Iijima, S. Nomura, K. Okumura, K. Takashima and K. Suzuki, Jpn. Kokai Tokkyo Koho JP 04041483,1992.

[7] H. Kano and S. Takahashi, "Shionogi Kenkyusho Nenpo," Shionogi Kenkyusho Nempo, Vol. 11, No. 1, 1961, pp. 1-3.

[8] G. Fengler, D. Arlt, K. Grohe, H. J. Zeiler and K. Metzger, “4H-1,4-Benzothiazin-Derivate," Ger. Offen. DE 3229125, 1984.

[9] T. Kachhee, V. Gupta, D. C. Gautam and R. Gupta, "Synthesis of 4H-1,4-Benzothiazine-1,1-dioxides (Sulfones) and Phenothiazine-5,5-dioxides (Sulfones)," Phosphorus, Sulfur, and Silicon and the Related Elements, Vol. 180, No. 10,2005 , pp. 2225-2234. doi:10.1080/104265090917790

[10] G. Kumar, V. Gupta, D. Gautam and R. Gupta, "Synthesis of Sulfones OF 4H-1,4-Benzothiazines and Phenothiazines," Phosphorus, Sulfur, and Silicon and the Related Elements, Vol. 179, No. 10, 2004, pp. 1941-1948. doi:10.1080/10426500490466931
[11] V. Molteni, X. He, J. Nabakka, K. Yang, A. Kreusch, P. Gordon, B. Bursulaya, I. Warner, T. Shin, T. Biorac, N. S. Ryder, R. Goldberg, J. Doughty and Y. He, "Identification of Novel Potent Bicyclic Peptide Deformylase Inhibitors," Bioorganic \& Medicinal Chemistry Letters, Vol. 14, No. 6, 2004, pp. 1477-1481. doi:10.1016/j.bmcl.2004.01.014

[12] K. Gupta, B. S. Rathore, R. Gupta, V. Gupta and M. Kumar, "Synthesis and Spectral Studies of 4H-1,4-Benzothiazine S,S-Dioxides (Sulfones)," Heterocyclic Communications, Vol. 9, No. 4, 2003, p. 381.

[13] T. S. Yokum, J. Alsina and G. Barany, "Solid-Phase Syntheses of Heterocycles Containing the 2-Aminothiophenol Moiety," Journal of Combinatorial Chemistry, Vol. 2, No. 3, 2000, pp. 282-292. doi: $10.1021 /$ cc9900854

[14] S. C. Schou, H. C. Hansen, T. M. Tagmose, H. C. M. Boonen, A. Worsaae, M. Drabowski, P. Wahl, P. O. G. Arkhammar, T. Bodvarsdottir, M.-H. Antoine, P. Lebrun and J. B. Hansen, "Synthesis and Pharmacological Evaluation of $4 \mathrm{H}-1,4$-Benzothiazine-2-carbonitrile 1,1-dioxide and N-(2-Cyanomethylsulfonylphenyl)acylamide Derivatives as Potential Activators of ATP Sensitive Potassium Channels," Bioorganic \& Medicinal Chemistry Letters, Vol. 13, No. 1, 2005, pp. 141-155. doi:10.1016/j.bmc.2004.09.051

[15] A. I. Gerasyuto, S. G. Zlotin and V. V. Semenov, "Synthesis of 2,3-Dihydrobenzothiazol-1,1-dioxide and 2,3Dihydro-1,4-benzothiazin-3-one Nitroderivatives from 2,4-Di- and 2,4,6-Trinitrobenzamides," Synthesis, Vol. 2, 2001, pp. 300-304. doi:10.1055/s-2001-10820

[16] F. Babudri, S. Florio, A. M. Vitrani and L. Di Nunno, "Synthesis of 4H-1,4-Benzothiazines via Lithiation Alpha to Sulphur of 2-Acylaminophenyl Alkyl Sulphides, Sulphoxides, and Sulphones," Journal of the Chemical Society, Perkin Transactions 1, Vol. 8, 1984, pp. 1899-1903. doi:10.1039/p19840001899

[17] Y. Ishikawa, Y. Terao, K. Suzuki, N. Shikano and M. Sekiya, "Cyclization of $\alpha$ - and $\beta$-Alkylthio-Substituted Amines Possessing Positively Charged Carbon at the Nitrogen. A New Synthetic Method for Thiazolidines, Thiomorpholines and Dihydro-1,4-benzothiazines," Chemical \& Pharmaceutical Bulletin, Vol. 32, No. 2, 1984, pp. 438-446. doi: $10.1248 / \mathrm{cpb} .32 .438$

[18] G. Pagani, "Ricerche sui Solfoni Ciclici. Nota V. 4H1,4-Benzothiazine-1,1-diossido," Gazzetta Chimica Italiana, Vol. 97, 1967, pp. 1804-1807.

[19] A. S. Angeloni and G. Pappalardo, "Benzothiazinone Diossidi e Loro Derivati," Gazzetta Chimica Italiana, Vol. 91, 1961, pp. 633-635.

[20] G. Fengler, D. Arlt and K. Grohe, "4H-1,4-Benzothiazin-derivate," Ger. Offen. DE 3229124, 1984.

[21] S. E. Lopez, J. Charris, N. Urdaneta and G. Lobo, "Synthesis of N-Aryl Substituted 4H-1,4-Benzothiazine 1,1Dioxide 2-Carboxylic Acid-Esters," Phosphorus, Sulfur, and Silicon and the Related Elements, Vol. 143, No. 1, 1998, pp. 53-61. doi:10.1080/10426509808045484

[22] S. E. Lopez, M. V. Godoy, N. Urdaneta and M. Rosales, "An Improved Procedure for the Preparation of N-Aryl 
Substituted 4H-1,4-Benzothiazine 1,1-Dioxide Derivatives," Phosphorus, Sulfur, and Silicon and the Related Elements, Vol. 156, No. 1, 2000, pp. 69-80. doi:10.1080/10426500008044994

[23] S. E. Lopez, J. Charris, N. Urdaneta, C. E. Canelon, J. Salazar, J. Herrera and J. E. Angel, "Unexpected Desulfonation $\mathrm{OF} \alpha$-Phenylsulfonyl Enaminoacrylates during Their Cyclisation to New N-Aryl 4H-1,4-Benzothiazine1,1-dioxides," Phosphorus, Sulfur, and Silicon and the Related Elements, Vol. 175, No. 1, 2001, pp. 87-97. doi:10.1080/10426500108040258

[24] S. E. Lopez, J. Salazar, O. Rebollo and J. Restrepo, “A Microwave Induced Cyclisation of $\alpha$-Phenylsulfonyl-enaminoacrylates for the Preparation of 4-Aryl-4H-1,4-benzothiazine 1,1-Dioxide Derivatives," Journal of Heterocyclic Chemistry, Vol. 42, No. 5, 2005, pp. 1007-1010. doi:10.1002/jhet.5570420541

[25] J. Charris, A. Barazarte, J. Dominguez and N. Gamboa, "Microwave-Assisted Synthesis of Quinolones and 4H1,4-Benzo-thiazine 1,1-Dioxides," Journal of Chemical Research, Vol. 1, No. 2, 2005, pp. 27-28. doi: $10.3184 / 0308234053431158$

[26] T. P. Culbertson, "Synthesis of 4H-1,4-Benzothiazine 1Oxide and 1,1-Dioxide. Analogs of Quinolone Antibacterial Agents," Journal of Heterocyclic Chemistry, Vol. 28, No. 7, 1991, p. 1701.

[27] G. M. Coppola and R. E. Damon, "Novel Heterocycles. 6. The Condensation of Ethyl o-Fluorobenzoyl Acetate with Cyclic Imino Ethers," Journal of Heterocyclic Chemistry, Vol. 17, No. 8, 1980, pp. 1729-1731. doi:10.1002/jhet.5570170818

[28] L. V. Ershov and V. G. Granik, "Lactams of Acetals and Acid Amides, 45. Synthesis of Condensed 2-Pyridones from Activated Amides, Lactams, and Lactones," Chemistry of Heterocyclic Compounds, Vol. 21, No. 7, 1985, pp. 771-774. doi:10.1007/BF00519144

[29] S.-I. Hirokami, T. Takahashi, M. Nagata and T. Yamazaki, "Rearrangements of Dewar 4-Pyrimidinones and 4-Methoxy-2-azetidinones. Reactions through Azetidinyl and Acyl Cations," Journal of Organic Chemistry, Vol. 52, No. 12, 1987, pp. 2455-2468. doi:10.1021/jo00388a022

[30] K. Sukata, "A Simple and Convenient Method for the Synthesis of Sulfones Using Polyethylene Glycols or Their Dialkyl Ethers as Solvents or Catalysts," Bulletin of the Chemical Society of Japan, Vol. 57, No. 2, 1984, pp. 613-614. doi:10.1246/bcsj.57.613

[31] H. Techer, M. Lavergne and M. Pesson, "Organic Syntheses without Solvent: Preparation of Sulfones and Dithioacetals," Synthesis, Vol. 1, 1987, pp. 56-59. doi:10.1055/s-1987-27843

[32] J. S. Grossert, P. K. Dubey, G. H. Gill, T. S. Cameron and P. A. Gardner, "The Preparation, Spectral Properties, Structures, and Base-Induced Cleavage Reactions of Some $\alpha$-Halo- $\beta$-ketosulfones," Canadian Journal of Chemistry, Vol. 62, No. 4, 1984, pp. 798-807. doi:10.1139/v84-133

[33] C. A. Zezza, M. B. Smith, B. A. Ross, A. Arhin and P. L. E. Cronin, "Reaction of Organolithium Reagents with Lactim Ethers: Preparation of Cyclic 2-Alkyl Imines or 2,2Dialkyl Amines," Journal of Organic Chemistry, Vol. 49, No. 23, 1984, pp. 4397-4399. doi:10.1021/jo00197a013

[34] J. P. Célérier, M. G. Richaud and G. Lhommet, "Imidoylation Reactions: A Simple Direct Synthesis of 3-Amino2-alkenoic Esters (ß-Enaminoesters)," Synthesis, Vol. 3, 1983, pp. 195-197. doi:10.1055/s-1983-30276 